\title{
Contribution à l'étude théorique et expérimentale des déversoirs à variation linéaire
}

\section{A contribution to theoretical and experimental research on linear variation weirs}

\author{
PAR R. HLAVEK \\ INGÉNIEUR DU GÚNIE RURAL EN SERVICE DÉTACHÉ \\ AUPRÈS DU BUREAU DE REGHERCHES GÉOLOGTQUES, GÉOPHYSIOUES ET MINĖ̀ES
}

\begin{abstract}
Les études théoriques tendant à dêterminer le profil d'un déversoir en mince paroi réalisant une loi de débit linéaire peuvent être classées en denx groupes; dans le premier groupe, le profil est défini par une fonction circulaire inverse, alors que, dans le second, il peut être représenté sous la forme: $\mathrm{y}=A / \mathrm{x}^{2}$.

En passant en revue ces diverses études, l'auteur en fait la comparaison et en démontre l'équivalence. Le problème de la "section équivalente » est ensuite étudié en détail, en particulier dans le cas de la section circulaire qui présente certaines propriétés remarquables. Des indications sont ensuite données en vne de faciliter le calcul des profils en $A / \mathrm{x}^{2}$.

$\mathrm{Du}$ point de vae expérimental, les résultals obtenus par l'auteur sont comparés à l'ensemble des données d'expérimentation obtenues dans ce domaine, et plus particulièrement à celles de P. Morin. En recherchant les explications de l'écart important, de l'ordre de $20 \%$, observé entre les valeurs des coefficients de débit trouvées par P. Morin et par l'auteur, ce dernier met en évidence le fait que cet écart aurait dû être encore plus important si les essais avaient été faits dans les mêmes conditions; ceci attire une fois de plus l'attention sur le danger qui s'attache a l'utilisation de déversoirs de mesure dans des conditions différentes de celles des essais.
\end{abstract}

\begin{abstract}
Theoretical research aimed at determining the shape of a weir plate with a linear discharge relationship can be classified in two distinct groups; in one, the ueir profile is determined by on inverse circular function and in the other, it can be represented by an expression of the form $\mathrm{y}=A / \mathrm{x}^{\underline{g}}$.

The author reviews and compares the various studies carried out on this subject and shows that they are equivalent. The "equivalent cross-section" problem is considered in detail, especially for a circular section, which has a certain number of remarlable properties. Indications are then given on houl to simplify the culculations for weir profiles of the form $\mathrm{y}=\mathrm{A} A \mathrm{x}$.

The author then compares his own experimental results with those obtained by other workers in this field, among which especially those of $P$. Morin, In attempting to find an explanation for the large discrepancy (about 20 per cent) between his and $P$. Morin's discharge coefficients, he shows that the difference would probably have been even greater, had both sets of tests been carried out under the same conditions. In this way, the anthor again draws attention to the danger of using gauging weirs at other conditions than those at which the!l have been tested.
\end{abstract}

\section{I. - ETUDES THÉORIQUES : historique, synthèse et compléments}

Après un rappel des divers travaux concernant les déversoirs à loi de variation linéaire, nous en faisons la comparaison, puis la synthèse. I'ne place particulière est accordée au problème de la «section équivalente» en complément de ces résultats.

\section{Les déversoirs dits "Sutro-Weir "}

Une élude de H. H. Sutro, parue en 1908 et dont nous n'avons pas la référence exacte, a été reprise en 1914 par E. A. Pratt [1], puis en 1936 par E. Soucek, H. E. Howe et F. T. Ma- 


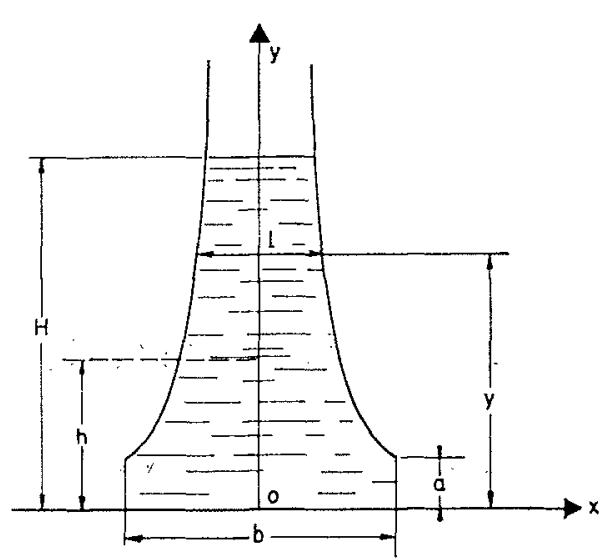

Fra. 1

vis [2], et en 1943 par E. G. Smith [3]. Plus récemment, R. A. Lincoln [4] a décrit trois déversoirs de ce type. L'ensemble des résultats peut se résumer ainsi : une loi de la forme $\mathrm{Q}=m \mathrm{H}-n$ correspond à un déversoir dont l'échancrure a une largeur $l$ définie par les relations :

$$
\begin{gathered}
l=b, \text { pour } y \leqslant a ; \\
l=b\left[1-\frac{2}{\pi} \cdot \operatorname{arctg} \sqrt{\frac{y-a}{a}}\right] \text { pour } y \geqslant a,
\end{gathered}
$$

avec les notations de la figure 1 .

La loi de débit peut s'écrire aussi :

$$
\mathrm{Q}=\theta b \sqrt{2} \overline{g a}(\mathrm{H}-a / 3),
$$

ce qui relie le couple de paramètres $(m, n)$ au couple $(a, b)$ par les deux égalités suivantes :

$$
a=3 \mathrm{n} / \mathrm{m} \quad \text { et } b=m / \theta \sqrt{2 g a}
$$

Considérant le cas d'une loi de débit quelconque : $\mathrm{Q}=f(\mathrm{H}), \mathrm{G}$. di Ricco a de son côté établi [5] l'équation générale de définition du profil satisfaisant à ladite loi, équation qui se présente ainsi :

$$
l=\frac{2}{\pi \sqrt{2 g}} \cdot \frac{d}{d y} \int_{0}^{y} \frac{d(\mathrm{Q} / \theta)}{d h} \cdot \frac{1}{\sqrt{y-h}} \cdot d h
$$

$h$ représente une variable d'intégration correspondant à l'intervalle $[0,+y]$, les autres notations étant les mêmes que précédemment.

Dans le cas où nous avons :

$$
\mathrm{Q}=m \mathrm{H}-n,
$$

le profil est ainsi défini (1) :

(1) Cet auteur a par ailleurs étudié des solutions approchées et de construction plus facile, consistant en contours polygonaux [7]; enfin le fonctionnement en déversoir noyé a été étudié lui aussi [8]. pour $y \leqslant a$,

$$
l=b
$$

et pour $y \geqslant a$,

$$
l=b\left[\frac{2}{\pi} \cdot \arcsin \sqrt{\frac{a}{y}}\right]
$$

les paramètres $\alpha$ et $b$ étant liés aux paramètres $m$ et $n$ comme plus haut et le débit pouvant naturellement se mettre encore sous la forme :

$$
\mathrm{Q}=\theta b \sqrt{2 g a}(\mathrm{H}-a / 3)
$$

Il est à peine besoin de souligner l'identité des résultats obtenus d'abord par $H$. H. Sutro et E. A. Prát, puis par G. di Ricco. Nous remarquerons cependant que, si les auteurs américains ont sans aucun doute donné les premiers ces résultats, les travaux de G. di Ricco sont certainement d'une portée théorique beaucoup plus générale, sa première étude [5] déjà citée ayant été d'ailleurs complétée par une autre [6] dans laquelle l'auteur examine le cas où $\theta$ est une fonction inconnue de $\mathrm{H}$.

\section{Les déversoirs dits en " tour Eiffel 》}

Ces déversoirs ont été particulièrement étudiés d'abord par P. A. Roubinet [13], - puis par P. Morin [9], auquel nous prenons d'ailleurs cette dénomination de déversoirs en «tour Eiffel ». Alors que ces auteurs se sont limités au cas particulier du déversoir à variation linéaire, L. Hugues [10] a étudié le cas plus général d'une loi de débit quelconque. Nous exposons ci-après l'essentiel des travaux de ces auteurs, en modifiant parfois les symboles utilisés dans les notes citées en références, afin de faciliter leur comparaison.

\section{$1^{\circ}$ Trayaux de P.A. Roubinet :}

Cet auteur est parti de la formule simplifiée du déversoir rectangulaire dont le débit est :

$$
\mathrm{Q}=m \mathrm{LH} \sqrt{2 g \mathrm{H}}
$$

$L$ étant la largeur dudit déversoir, H la charge et $m$ le coefficient de contraction. Considérant alors la section $\Omega$ de la veine liquide à la sortie du déversoir, et négligeant « pour simplifier, le léger abaissement de la veine au franchissement du seuil », P.A. Roubinet écrit (1) sous la forme :

$$
\mathrm{Q}=m \Omega \sqrt{2 g \mathrm{H}}
$$


Nous avons donc par définition :

$$
\mathrm{Q}=k \mathrm{H},
$$

et l'auteur décide d'appliquer «par analogie» l'équation (2); nous déduisons aussitôt de l'identification des deux valeurs de $Q$ les relations suivantes :

$$
\Omega=\alpha \sqrt{\mathrm{H}}
$$

et aussi :

$$
k=m \propto \sqrt{2 g}
$$

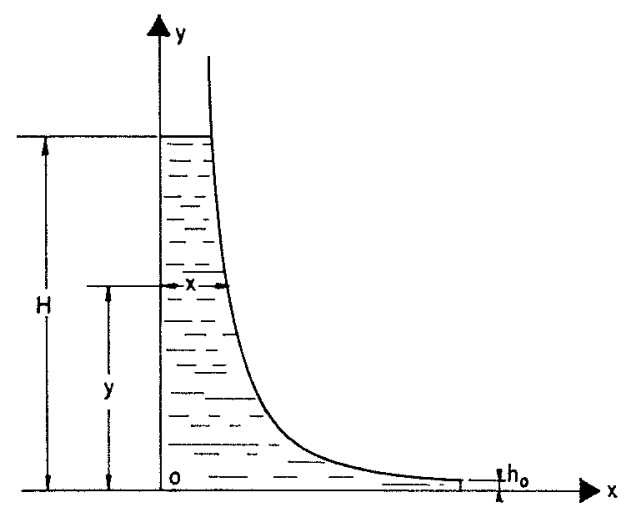

FrG. 2

Si $x=f(y)$ est l'équation du profil, et $\mathrm{F}(y)$ la primitive de $f(y)$, nous aurons $\mathrm{F}(\mathrm{H})=\Omega$; dans ces conditions, nous trouvons

d'où :

$$
f(\mathrm{H})=\alpha / 2 \sqrt{\mathrm{H}}
$$

$$
y=\frac{k^{2}}{8 m^{2} g} \cdot \frac{1}{x^{2}}
$$

Pour comparer ces résultats aux précédents, nous devons considérer le profil symétrique, et non plus seulement' le demi profil de P. A. Roubinet; il suffit pour cela de poser

$$
f(\mathrm{H})=\alpha / 4 \sqrt{\mathrm{H}}(\text { au lieu de } \alpha / 2 \sqrt{\mathrm{H}})
$$

d'où

$$
y=\frac{k^{2}}{32 m^{2} g} \cdot \frac{1}{x^{2}}
$$

\section{$2^{\circ}$ Travaux de P. Morin :}

Cet auteur admet que l'écoulement par le déversoir peut être assimilé à celui qui se ferait à travers un orifice dont le bord supérieur serait arasé au niveau $\mathrm{H}$ de la retenue. Il existe donc deux fonctions, l'une $y(x)$ représentant l'orifice assimilé au déversoir, l'autre $z(x)$ représentant l'échancrure. P. Morin admet que ces deux cour-

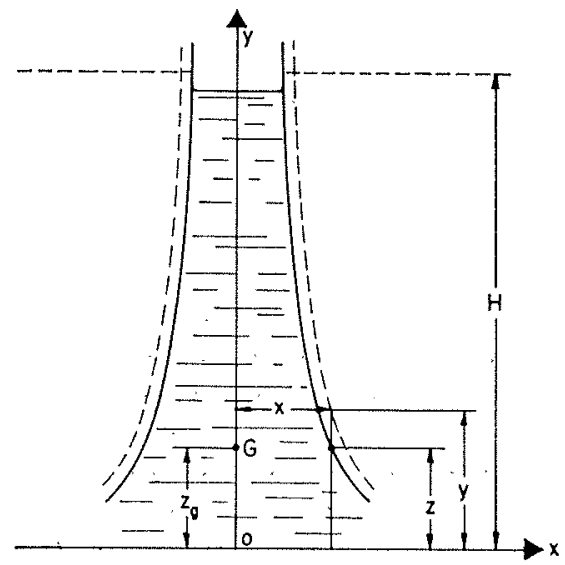

FIg. 3

bes sont probablement de la même famille, ce qui l'autorise à aborder le problème par la détermination de $y(x)$, plus facile. Le raisonnement est alors le suivant : si $\Omega$ est la surface mouillée, $m$ le coefficient de contraction et $z_{y}$ la cote du centre de gravité de cette surface, le débit est donné par la relation

$$
\left.\mathrm{Q}=m \Omega \sqrt{2 g(\mathrm{H}}-z_{l}\right) \# m \Omega \sqrt{2 g\left(\mathrm{H}-y_{\eta}\right)}
$$

Or nous avons :

$$
\Omega=2 \int_{0}^{\mathrm{H}} x(y) d y
$$

et

$$
y_{g}=\int_{0}^{\mathrm{H}} x(y) y d y / \int_{0}^{\mathrm{I}} x(y) d y
$$

et nous posons $\Omega=2 \mathrm{~S}$ d'une part, $y_{g}=\mathrm{V} / \mathrm{S}$ d'autre part. Si l'on introduit la condition

$$
\mathrm{Q}=k \mathrm{H},
$$

il vient finalement

$$
\mathrm{Q}=k \mathrm{H}=2 \mathrm{mS} \sqrt{2 g(\mathrm{H}-\mathrm{V} / \mathrm{S})}
$$

d'où l'on tire, en revenant à $y$ et en posant $\mathrm{C}=8 g \mathrm{~m}^{2} / \mathrm{k}^{2}$, l'équation différentielle

$$
y^{2} d \mathrm{~S} / d y-2 y \mathrm{~S}+\mathrm{CS}^{3}=0 ;
$$

dans les conditions du problème, la solution de cette équation différentielle est :

$$
y=3 k^{2} / 64 m^{2} g x^{2}
$$

En fait, la relation (1) doit être corrigée pour tenir compte, d'une part, du fait que la vitesse moyenne $v_{m}$ n'est pas égale à $\sqrt{2 g\left(H-y_{g}\right)}$, vitesse au centre de gravité, mais à

$$
\beta \sqrt{2 g\left(\mathrm{H}-y_{y}\right)},
$$

avec $\beta<1$, et, d'autre part, du fait que le débit 
n'a pas pour expression: $Q=m \Omega v_{m}$, mais plutòt $Q=\theta \Theta v_{m}, \theta$ ćtant le coefficient de débit, produit du coefficient de contraction $m$ par le coefficient de vitesse; on passe alors de (7) à

$$
y=\frac{3 k^{2}}{64 \beta^{2} \AA^{2} g} \cdot \frac{1}{x^{2}}
$$

Il est intéressant de comparer ces résultats à ceux obtenus par P. A. Roubinet. Remarquons tout d'abord que les profils définis par ces deux auteur's seront équivalents, si les valeurs de $x, y$ et $k$ sont les mêmes. Cette équivalence n'est possible qu'en admettant que les coefficients de contraction $m$ sont différents; soit $m^{\prime}$ le coefficient figurant dans la définition de P. A. Roubinet et $m$ celui défini par M. Morin, l'on doit alors avoir : $m^{\prime}=m \sqrt{2 / 3}$. Si nous écrivons enfin que les deux définitions du débit sont équivalentes, c'est-à dire que :

$$
\mathrm{Q}=m^{\prime} \Omega \sqrt{ } 2 \overline{\mathrm{HH}}=m \Omega \sqrt{2 g\left(\overline{H-y_{g}}\right),}
$$

il vient

$$
\left(\mathrm{H}-\mathrm{y}_{g}\right)=2 \mathrm{H} / 3 .
$$

Or, il est facile de démontrer que les courbes en $y=\mathrm{A} / x^{2}$ sont telles que cette relation est toujours vérifiée. Le fait d'écrire, avec P. A. Roubinet, que l'on a $Q=m \Omega \sqrt{2 g \mathrm{H}}$, revient en quelque sorte à supposer le problème résolu; le calcul de P. Morin est donc seul rigoureux.

\section{$3^{\circ}$ Travaux de L. Hugues:}

Assimilant lui aussi l'écoulement par le déversoir à l'écoulement par un orifice, cet auteur pose, conformément à la formule dite de Torricelli, que la vitesse dans le filet liquide amenant l'eau à l'élément vertical, dans le plan du déversoir, et d'aire $d \Omega=2 x d y$ est déterminée par la distance $\mathrm{H}-y$ qui sépare de la surface libre l'élément considéré; cette vitesse ayant

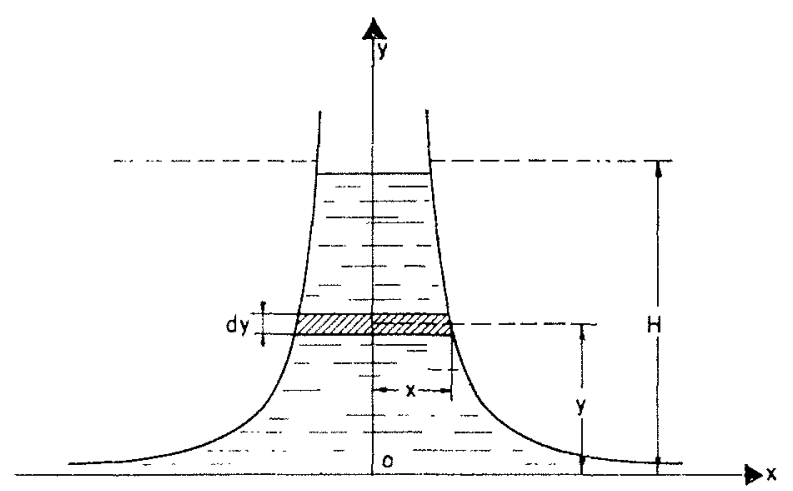

FIG. 4 alors pour valeur $\sqrt{2 g(H-y)}$, le débit a pour expression :

$$
\mathrm{Q}=2 \sqrt{2 g} \int_{0}^{\mathrm{H}} x \sqrt{\mathrm{H}-y} d y
$$

$x$ étant la fonction $f(y)$ à déterminer.

L'auteur étudie le cas général d'une loi de débit quelconque $\mathrm{Q}=\lambda(\mathrm{H})$ qu'il met sous la forme

$$
\mathrm{Q}=2 \vee^{\prime} \overline{2 g} \Phi(\mathrm{H})
$$

Le rapprochement des relations (1) et (2) domne l'équation intégrale linéaire

$$
\Phi(\mathrm{H})=\int_{0}^{\mathrm{H}} f(y) \sqrt{\mathrm{H}-y} d y
$$

dont la solution est :

$$
f(y)=\frac{2}{\pi} \cdot\left[\frac{\Phi^{\prime}(0)}{\sqrt{y}}+\int_{0}^{y} \frac{\Phi^{\prime \prime}(\mathbf{H})}{\sqrt{y-\mathrm{H}}} \cdot d \mathrm{H}\right]
$$

Il étudie alors plus spécialement le cas de

$$
\Phi(\mathrm{H})=\mathrm{KH}^{n},
$$

$n>1 / 2$ désignant un nombre positif; dans le cas particulier de $n=1$, il aboutit à la relation ${ }^{(2)}$ :

$$
x=2 \mathrm{~K} / \pi \vee \bar{y}
$$

Si nous considérons que nous avons d'une part : $Q=k H$, et d'autre part $Q=2 \sqrt{2 g} \Phi(H)$ (équation 2), (5) devient alors:

$$
x=k / \pi \sqrt{2 g_{y}}
$$

En fait, les hypothèses de base de L. Hugues doivent être complétées afin de tenir compte, d'une part, des contractions à la traversée de la paroi et, d'autre part, des forces de frottement. Le débit élémentaire considéré par l'auteur doit alors être multiplié par le «coefficient de débit» D, $(0<1)$, et $(3)$ devient :

$$
\Phi(\mathrm{H})=\int_{0}^{\mathrm{H}} \theta f(y) \sqrt{\mathrm{H}-y} d y
$$

Si $\theta$ est une constante, les calculs restent valables dans le détail et nous pouvons tirer de (6) la relation suivante :

$$
y=\frac{k^{2}}{2 \pi^{2} g \theta^{2}} \cdot \frac{1}{x^{2}}
$$

(2) Cette relation peut se déduire directement de la solution générale (4), puisqu'avec $\Phi(\mathrm{H})=\mathrm{KH}$ nous avons $\Phi^{\prime}(0)=\mathrm{K}$ et $\Phi^{\prime \prime}(\mathrm{H})=0$. 
$\mathrm{Si}$, au contraire, $\theta$ est une fonction de $y$, eile est inconnue, tout comme $f(y)$, et l'équation intégrale linéaire n'est plus soluble.

4" Identité des Résultats de P. Morin E DE L. Hugues :

Nous précisons que, conformément à ce que nous avons exposé ci-dessus, les résultats obtenus par P. A. Roubinet doivent être confondus avec ceux de P. Morin. Il reste donc à comparer ces derniers à ceux de L. Hugues, c'est-à-dire à comparer les équations

et

$$
y=\frac{3 k^{2}}{64 g^{2} \theta^{2}} \cdot \frac{1}{x^{2}} \text { de P. Morin, }
$$

$$
y=\frac{k^{2}}{2 g \pi^{2} \eta^{2}} \cdot \frac{1}{x^{2}} \text { de L. Hugues. }
$$

En fait les conditions du problème étant les mêmes et les raisonnements tous deux rigoureux, nous pouvons affirmer a priori que ces deux résultats sont identiques. Nous en déduisons immédiatement la relation $\beta=\pi \sqrt{3} / 4 \sqrt{2}$, que nous croyons cependant intéressant de démontrer en revenant quelque peu sur la définition du coefficient $\beta$ : soit un orifice de section $Q$, de largeur variable $b(h)$, placé en paroi latérale verticale; dans une tranche d'aire :

$$
d \Omega=b(h) d h,
$$

la vitesse est constante et égale à $v==\sqrt{2 g h}$ (3).

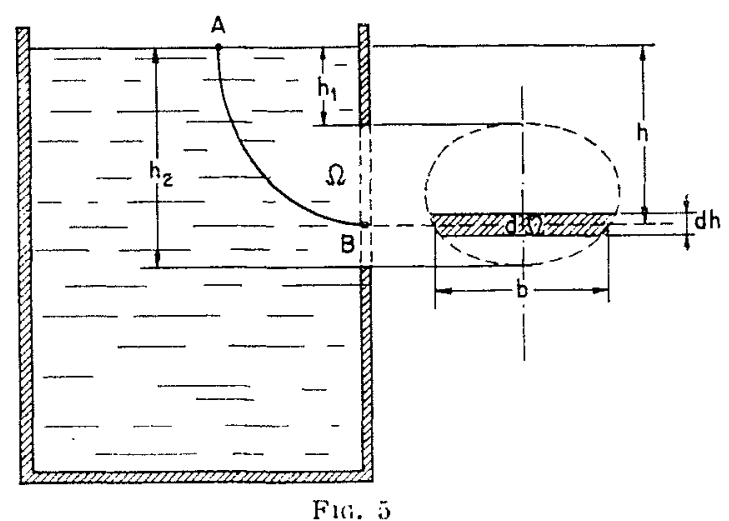

Dans ces conditions le débit total est :

$$
\mathrm{Q}=\int_{\Omega} v d \Omega=\int_{h_{1}}^{h_{2}} b(h) \sqrt{2 g h} d h
$$

La vitesse moyenne dans la section est égale à

$$
v_{m}=\beta \sqrt{2 g} h_{y},
$$

(3) En fait, et nous l'avons déjà signale à propos des résultats de $L$. Hugues, le coeffeient de débit $\theta$ intervient. Si $\theta$ est constant, quel que soit $y$, les résultats sont inchangés. $h_{g}$ étant la charge sur le centre de gravité $\mathrm{G}$ de l'aire $Q$, et l'on écrit (3);

$$
\mathrm{Q}=\beta \Omega \sqrt{2 g h_{l}}
$$

L'on en déduit :

$$
\beta=\frac{1}{\Omega \sqrt{h_{g}}} \cdot \int_{h_{1}}^{h_{2}} b(h) \sqrt{h} d h,
$$

ce qui, dans le cas qui nous intéresse, devient, avec les notations de la figure 6 :

$$
\beta=\frac{1}{\Omega \sqrt{h_{y}}} \cdot \int_{0}^{\mathrm{H}} 2 x \sqrt{h} d h
$$

or, nous avons :

$$
x=\sqrt{\mathrm{A} /(\overline{\mathrm{H}}-h)}, \quad \Omega=4 \sqrt{\mathrm{AH}}
$$

et

Finalement :

$$
h_{y}=2 \mathrm{H} / 3
$$

$\beta=\frac{\sqrt{3 / 2}}{2 \mathrm{H}} \int_{0}^{\mathrm{H}} \sqrt{\frac{h}{\mathrm{H}-h}} \cdot d h=\frac{\pi}{4} \cdot \sqrt{\frac{3}{2}}$

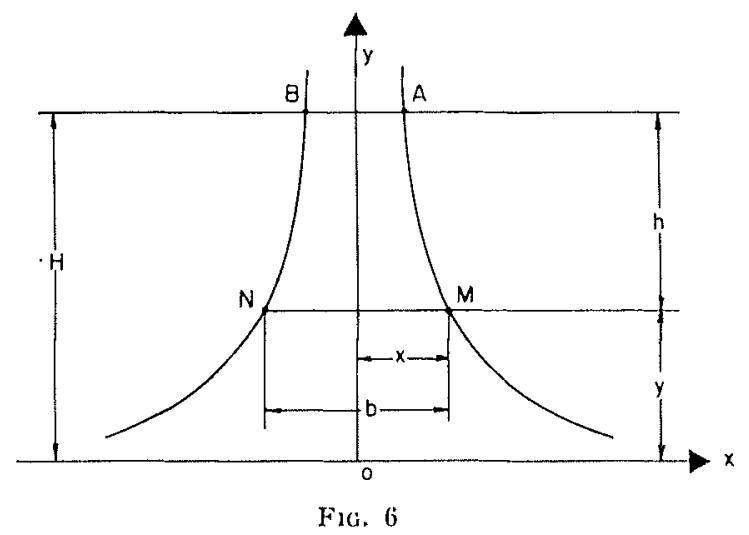

Remarquons enfin que le coefficient $\beta$ est dans ces conditions indépendant à la fois non seulement de la charge $H$ mais aussi du paramèlre $A$. C'est là une propriété remarquable, que nous aurons d'ailleurs à utiliser plus loin.

\section{Comparaison des travaux de G. di Ricco et L. Hugues}

L’équation générale établie par G. Di Ricco donne, appliquée au cas d'une loi de débit:

$$
\mathrm{Q}=m \mathrm{H}-n,
$$

un déversoir du type que nous avons appele 
Sutro-Weir et nous avons à ce propos souligné l'identité des divers résultats. 'Si au contraire nous posons, dans ladite équation $\mathrm{Q}=k h$, la largeur $l$ de l'échancrure à la cote $y$ est alors définie par l'équation plus simple

$$
l=2 x=\frac{2}{\pi \sqrt{2 g}} \cdot \frac{d}{d y} \int_{0}^{y} \frac{k}{\theta \sqrt{y-h}} \cdot d h,
$$

d'où l'on tire :

$$
y=\frac{k^{2}}{2 g \pi^{2} \theta^{2}} \cdot \frac{1}{x^{2}}
$$

Or, c'est exactement ce derniè résultat que nous avons trouvé en faisant: $\mathrm{Q}=k \mathrm{H}$ dans l'équation générale de L. Hugues: Nous pouvons donc dire, compte tenu des identités de résultats déjà démontrées, que l'ensemble des relations établies par les divers auteurs sont théoriquement équivalentes, sous réservè des modifications que nous avons signalées au passage.

Il importe toutefois de remarquer que cette identité vaut dans le cas où nous avons : $\mathrm{Q}=k \mathrm{H}$, ce qui correspond à des profils asymptotiques au seuil, que nous avońs appelés «en Tour Eiffel $\gg$; en raison des difficultés matérielles, la partie asymptotique ne peut être réalisée et l'on aboutit, comme nous le précisons ci-après, à des profils non asymptotiques au seuil, réalisant une loi de débit du type : $\mathrm{Q}=m H-n$, tout en conservant une définition de profil en $\mathrm{A} / x^{2}$, et non en $\operatorname{Arc} \sin \vee \overline{a / y}$.

\section{Les déversoirs en " tour Eiffel » : problème de la section équivalente}

La partie asymptotique de la courbe $y=A / x^{2}$ étant difficilement réalisable pour de simples questions d'usinage, une première solution fut proposée par P.A. Roubinet, dans l'étude déjà citée, solution qui était de ne faire partir le profil qu'à partir d'une certaíne hauteur $h_{0}$, prise volontairement faible (par exemple égale au $1 / 100^{\circ}$ de la charge maximale), la largeur $x_{0}$ de l'échancrure à cette cote étant maintenue constante pour $y$ compris entre $h_{0}$ et 0 ; les résultats des essais ont montré qu'alors la loi de débit était de la forme: $\mathrm{Q}=k \mathrm{kH}$ - Cte; cette solution présente l'avantage de la simplicité, mais elle n'est qu'une approximation, d'autant meilleure naturellement que $h_{0}$ est plus faible.

La deuxième solution est due à P. Morin [9] qui propose de remplacer toute la partie de la courbe située au-dessous d'uné cote $y=y_{0}$ par une \& section équivalente», ayant même aire et même centre de gravité que la partie remplacée, c'est-à-dire fournissant le même débit. En toute rigueur, les coefficients de débit $\theta$ et les coefficients $\beta$ devraient eux aussi être égaux; ces conditions supplémentaires ne pouvant toutefois être introduites dans nos calculs, il ne sera question dans les lignes qui suivent que des conditions fondamentales de coïncidence des centres de gravité et d'égalité des aires.

\section{$1^{\circ}$ Section Équivalente Rectangulaire.}

Si nous considérons le profil interrompu en $A$ et $B$, points définis par la droite d'équation $y=y_{0}$, deux cas sont possibles, selon que ces points $A$ et $B$ sont sommets du rectangle, ou seulement sur l'arête supérieure.

1.1-A et $B$ sur l'arête supérieure du rectangle (cas de la figure 7).

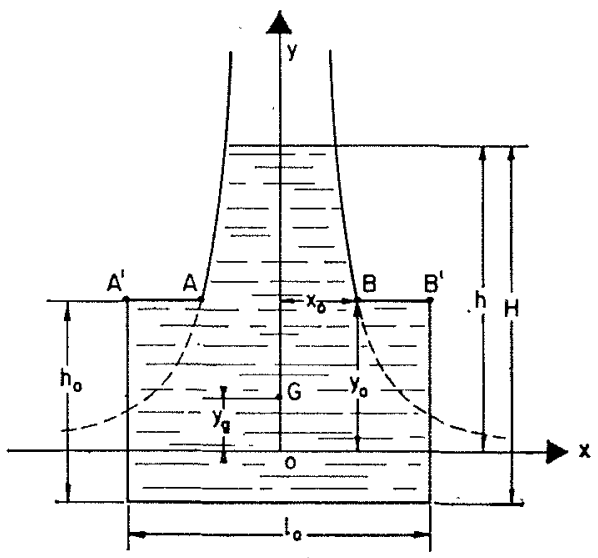

FIG. 7

Dans ce cas; nous nous imposons deux conditions, pour deux paramètres à déterminer; nous devons donc toujours pouvoir déterminer un rectangle équivalent, quelle que soit la valeur de $y_{0}$.

$P$. Morin a démontré [9] que ce rectangle a pour caractéristiques $h_{0}=4 y_{0} / 3, \quad l_{0}=3 x_{0}$. d'où la figure 7 .

Nous pensons qu'il n'est pas inutile de faire la remarque suivante: la loi $\mathrm{Q}=k h$ peut aussi s'écrire $\mathrm{Q}=\boldsymbol{k}\left(\boldsymbol{H}-y_{0} / 3\right)$, 'que nous pouvons identifier à $\mathrm{Q}=m \mathrm{H}-n$, à condition que

$$
m \cong k \quad \text { et } n \cong k y_{0} / 3 \text {. }
$$

Si nous rappelons par ailleurs que la loi

$$
Q=m \mathrm{H}-n
$$

s'applique à un Sutro-Weir de paramètres $a$ et $b$ tels que l'on ait $a=3 n / m$ 
et

$$
b=m / \theta \sqrt{2 g a},
$$

nous pouvons conclure que ce déversoir satisfera à la relation

$$
\mathrm{Q}=k\left(H-y_{0} / 3\right)
$$

$\grave{a}$ condition que $a=y_{0}$ et

$$
b=k / \theta \sqrt{2 g !_{0}} .
$$

Ces dimensions. du rectangle sont à rapprocher des valeurs $h_{0}$ et $l_{0}$, et il est facile de vérifier que l'on a :

et

$$
h_{0}=4 a / 3
$$

$$
l_{0}=b \sqrt{27 / 32}
$$

la relation entre les aires des deux rectangles s'en déduit aussitôt.

1.2 - A et $B$ sommets du rectangle (cas de la figure 8 ).

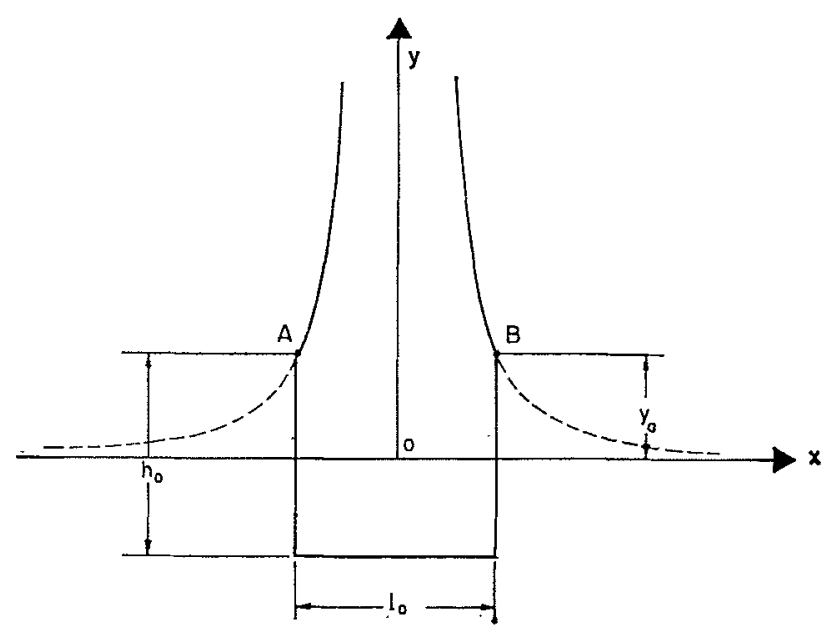

Fia. 8

Cette condition qui vient s'ajouter aux conditions fondamentales rend le problème impossible d'une manière quelconque. A et $B$ étant sommets, cela permet d'écrire que la largeur $l_{0}$ du rectangle est égale à $2 x_{0}$, d'où $\Omega_{0}=2 x_{0} h_{0}$. Par ailleurs, et avec $y=A / x^{2}$, nous avons :

$$
\Omega_{0}=4 \mathrm{~A} / x_{0}
$$

En égalant ces deux valeurs, nous trouvons $h_{0}=2 y_{0}$, ce qui implique que le rectangle soit centré sur l'axe $\overrightarrow{O x}$, alors que la surface $\Omega_{0}$ ne peut qu'avoir son centre de gravité au-dessus de cet axe. Ce cas de figure est donc impossible.

\section{$2^{\circ}$ Section ḱquivalente circulaire.}

Ici encore nous avons deux cas possibles selon que le cercle passe ou non par les points $A$ et $B$.

\section{1 - Cas où le cercle ne passe pas par $A$ et B :}

Le cercle étant défini par son rayon $R$ et la cote $a$ de son centre, nous pourrons toujours trouver un cercle équivalent, puisque nous n'imposons que les deux conditions fondamentales. Plusieurs cas de figure sont cependant possibles selon les valeurs relatives de $y_{0}$ et de $a$.

2.1.1. - Cas où $y_{0}>a$ (cas de la figure 9): en adoptant les notations de la figure 9 et en rappelant que la distance $\mathrm{CG}$, séparant le centre $\mathrm{C}$ du cercle du centre de gravité $G$ de la surface $S$ limitée par le cercle et la droite $y=y_{0}$, est :

$$
\mathrm{CG}=-2 \mathrm{R}^{3} \sin ^{3} \theta / 3 \mathrm{~S}
$$

les trois inconnues $a, \mathrm{R}$ et $\theta$ sont liées par les trois équations ci-dessous :

$$
\begin{gathered}
a+\mathrm{R} \cos \theta=y_{0} \\
\mathrm{R}^{2}[(\pi-\theta)+\sin \theta \cos \theta]=\mathrm{S} \\
=4 \sqrt{\mathrm{A} y_{0}}=4 \mathrm{~A} / x_{0} \\
a-2 \mathrm{R}^{3} \sin ^{3} \theta / 3 \mathrm{~S}=y_{0} / 3
\end{gathered}
$$

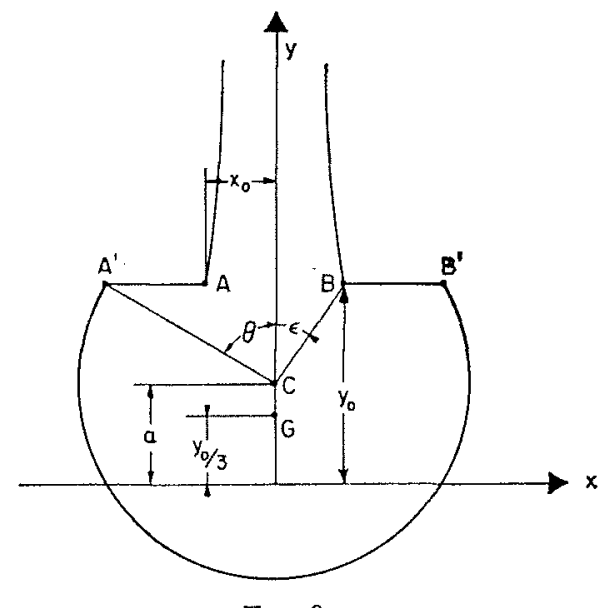

FIG. 9

Ce système peut être réduit à une seule équation :

$\sqrt{\frac{A}{2 x_{0}{ }^{3}}}=\frac{3 \cos \theta[2(\pi-\theta)+\sin 2 \theta]+4 \sin ^{3} \theta}{[2(\pi-\theta)+\sin 2 \theta]^{3 / 2}}$

Si nous considérons la fonction

$$
y=\frac{3 \cos \theta[2(\pi-\theta)+\sin 2 \theta]+4 \sin ^{3} \theta}{[2(\pi-\theta)+\sin 2 \theta]^{3 / 2}}
$$


une étude rapide nous permet de voir qu'elle présente l'allure de la figure 10

pour l'intervalle : $0<0<+\pi / 2$,

seul intéressant.

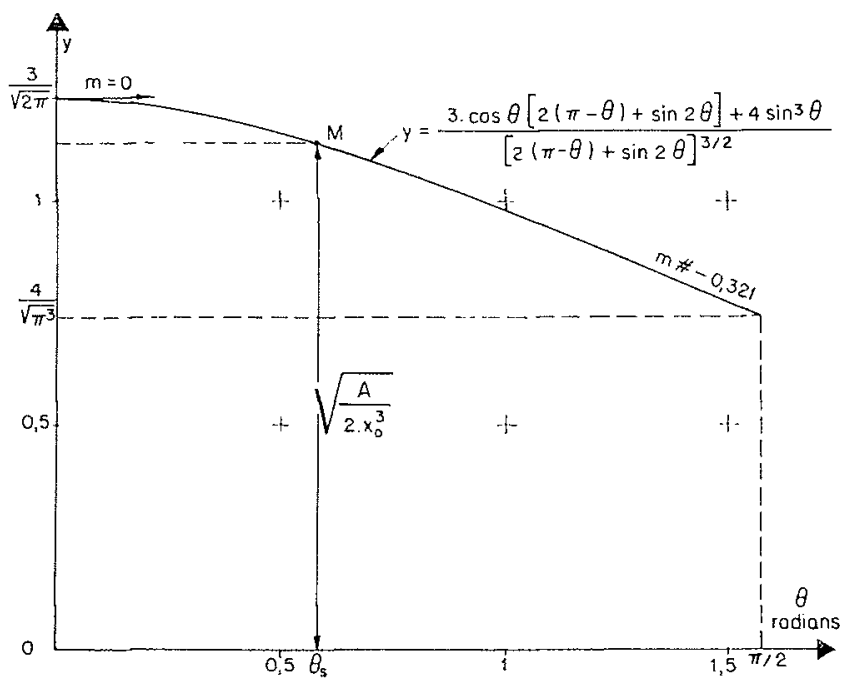

Fig. 10,

Connaissant donc $x_{0}$ (4) valeur fixée à l'avance, l'on peut trouver une solution $\theta_{\mathrm{s}}$. Il importe pour cela que les deux courbes

et

$$
y=\sqrt{\mathrm{A} / 2 x_{0}^{3}}
$$

$$
y=\frac{3 \cos \theta[2(\pi-\theta)+\sin 2 \theta]+4 \sin ^{3} 0}{[2(\pi-\theta)+\sin 2 \theta]^{3,2}}
$$

se coupent, ce qui donne l'inégalité :

$$
\sqrt[3]{\pi A / 9} \leqslant x_{0} \leqslant \pi \sqrt[3 / 2 A]{2} 4
$$

Nous pouvons dire dans ces conditions que :

- pour $x_{0}=\sqrt[3]{\pi \mathrm{A} / 9}$, nous avons $\theta=0$, ce qui est impossible;

- pour $x_{0}=\pi \sqrt[3]{2 \mathrm{~A}} / 4$, nous avons $\theta=+\pi / 2$, cas étudié plus loin $(\$ 2.1 .3$.$) ;$

- pour $\sqrt[3]{\pi \mathrm{A} / 9} \leqslant x_{0} \leqslant \pi \sqrt[3 / 2 \mathrm{~A} / 4]{ }$, la recherche se poursuit ainsi : $x_{0}$ étant donné et $\theta_{\mathrm{S}}$ venant d'être déterminé, $R$ peut être calculé en faisant $\theta=\theta_{\mathrm{s}}$ dans l'équation (2), puis a peut être calculé soit à l'aide de l'équation (3), soit plus simplement à l'aide de l'équation (1). Le calcul de $\theta$ n'est donc gu'un intermédiaire.

2.1.2- Cas où $y_{0}<a$ (cas de la figure 11): en adoptant les notations de la figure 11, et en rappelant que l'on a encore

$$
\mathrm{CG}=-2 \mathrm{R}^{3} \sin ^{3} \theta / 3 \mathrm{~S}
$$

(4) Il est certain que la solution est immédiate si au contraire c'est $\theta$ qui est fixé à l'avance [ct. équation (4)].

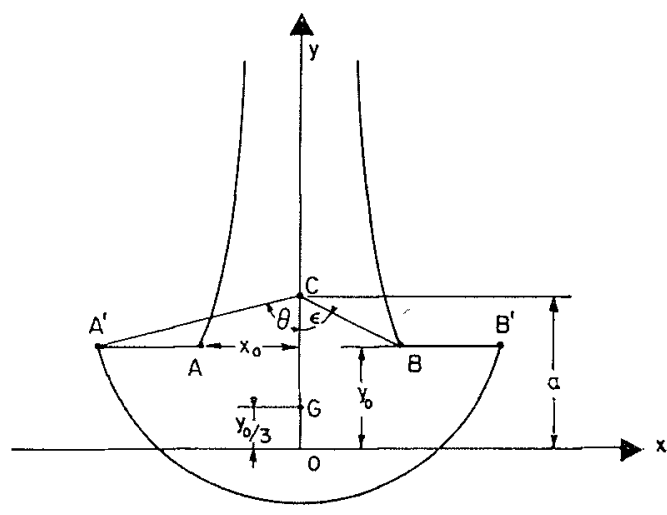

FIG. 11

les trois inconnues $a, R$ et 0 sont liées par les trois équations ci-dessous :

$$
a-y_{0}=\mathrm{R} \cos 0
$$

$\mathrm{R}^{2}[\theta-\sin \theta \cos \theta]=\mathrm{S}=4 \sqrt{\mathrm{A} y_{0}}=4 \mathrm{~A} / x_{0}$

$$
a-2 \mathrm{R}^{3} \sin ^{3} 0 / 3 \mathrm{~S}=y_{0} / 3
$$

De même qu'au $\$ 2.1 .1$ ce système peut être ramené à une seule équation

$$
\sqrt{\frac{\mathrm{A}}{2 x_{0}{ }^{3}}}=\frac{4 \sin ^{3} \theta-3 \cos \theta[2 \theta-\sin 2 \theta]}{[2 \theta-\sin 2 \theta]^{3 / 2}}
$$

La solution $\theta\left(y_{0}\right)$ est donnée par l'intersection des deux courbes:

et

$$
y=\sqrt{\mathrm{A} / 2 x_{0}^{3}}
$$

$$
y=\frac{4 \sin ^{3} \theta-3 \cos \theta[2 \theta-\sin 2 \theta]}{[2 \theta-\sin 2 \theta]^{3 / 2}},
$$

cette dernière équation présente l'allure de la figure 12 pour l'intervalle $0<\theta<+\pi / 2$, seul intéressant.

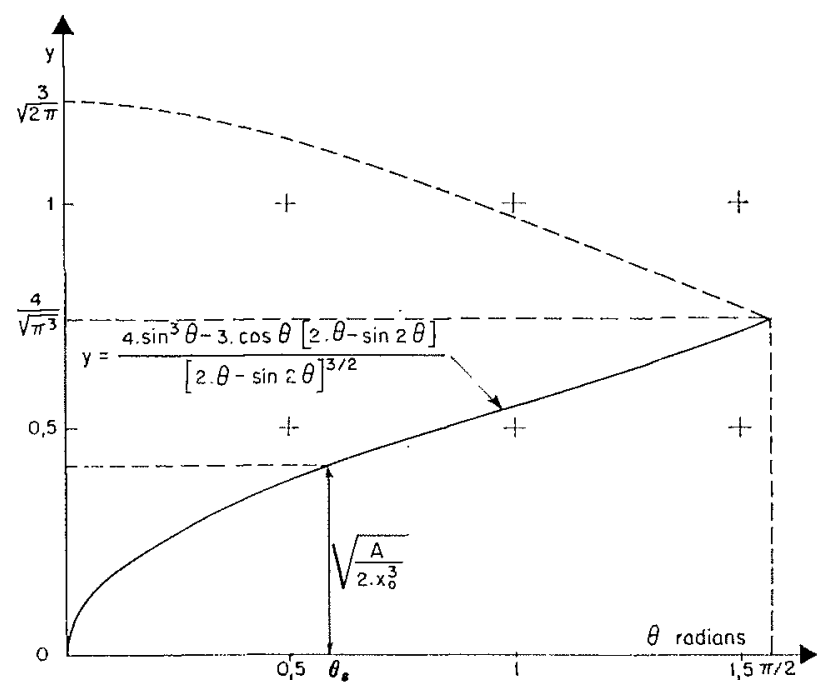

FIG. 12 
La valeur de $y_{0}$, ou de $x_{0}$, étant fixée, il y aura une solution $\theta_{\mathrm{S}}$ à condition que les deux courbes se coupent, ce qui donne l'inégalité

$$
\pi \sqrt[3]{2 \mathrm{~A}} / 4 \leqslant x_{0} \leqslant+\infty
$$

Nous pouvons dire dans ces conditions que :

- pour $x_{0}=\pi \sqrt[3]{2 A} / 4$, nous avons encore

$$
\theta=+\pi / 2 \text {, }
$$

cas étudié $\$ 2.1 .3$

- pour $x_{0} \rightarrow+\infty$, nous retrouvons $0=0$, mais ceci n'est plus impossible comme dans le cas de figure 9, et correspond au cas limite du cercle qui tend à devenir l'axe $\overrightarrow{O x}$;

- pour $\pi \sqrt[3]{2 \mathrm{~A} / 4}<x_{0}<+\infty$, $0_{\mathrm{s}}$ étant alors détermine, il reste à calculer $R$ à partir de $\left(\boldsymbol{2}^{\prime}\right)$ et $a$ à partir de $\left(\boldsymbol{1}^{\prime}\right)$.

2.1.3 - Cas où $y_{0}=a$ : la figure peut se déduire des figures 9 ou 11 indifféremment, en y faisant $\theta=+\pi / 2$; aux $\S \S 2.1 .1$ et 2.1.2, nous avons trouvé ce cas limite, correspondant à

$$
x_{0}=\pi \sqrt[3]{2 \mathrm{~A}} / 4 \text {. }
$$

Il est cependant possible d'établir directement ces résultats :

- tout d'abord la surface $\mathrm{S}$ prend la valeur particulière $\mathrm{S}=\pi \mathrm{R}^{2} / 2$; or nous avons toujours $\mathrm{S}=4 \mathrm{~A} / x_{0}$ d'où, par rapprochement : $\mathrm{R}^{2}=8 \mathrm{~A} / \pi x_{0}$;

- par ailleurs en faisant : $\sin 0=1$ et $y_{0}=-a$ dans (3) ou (3'), l'on trouve

$y_{0}=\mathrm{R}^{3} / \mathrm{S}=2 \mathrm{R} / \pi \quad$ d'où $: \mathrm{R}=\pi \mathrm{A} / 2 x_{0}{ }^{2}$;

- en égalant les deux valeurs de $\mathrm{R}$ ainsi trouvées, nous avons :

$x_{0}=\pi \sqrt[3 / 2 \mathrm{~A} / 4]{ } \quad$ et $\mathrm{R}=4 \sqrt[3]{2 \mathrm{~A}} / \pi=16 x_{0} / \pi^{2}$

2.1.4-Conclusions : nous pouvons dire que :

- pour $x_{0}>\sqrt[3]{\pi \mathrm{A} / 9}$, nous avons toujour's des solutions :

a) pour $x_{0}$ quelconque dans l'intervalle

$$
|+\infty, \pi \sqrt[3]{2 \mathrm{~A} / 4}|
$$

il existe à chaque fois une solution correspondante $(\$ 2.1 .2)$ et nous sommes dans le cas de la figure 11 ;

b) pour $x_{0}=\pi \sqrt[3]{2 \mathrm{~A}} / 4$, tout est déterminé $\left(\mathrm{R}=4 \sqrt[3]{2 \mathrm{~A}} / \pi\right.$ et $\left.a=y_{0}\right)(\$ 2.1 .3)$.

c) pour $x_{0}$ quelconque dans l'intervalle

$$
|\pi \sqrt[3]{2 \mathrm{~A}} / 4, \sqrt[3]{\pi \mathrm{A} / 9}|
$$

il existe à chaque fois une solution correspondante $(\$ 2.1 .1)$ et nous sommes dans le cas de la figure 9 .

- pour $x_{0}<\sqrt[3]{\pi \mathrm{A} / 9}$, il n'y a pas de solution.

Remarquons toutefois qu'une certaine imprécision demeure pour $x_{0} \# \sqrt[3 / \pi / 9]{\pi /}$ nous venons de dire que, pour $x_{0}>\sqrt[3]{\pi \mathrm{A} / 9}$, nous avions toujours une solution, mais il y a lieu de considérer que, si $x_{0}$ est à peine supérieur à cette valeur limite correspondant à $0=0$ (fig. 9), la valeur $\theta_{\mathrm{s}}$ correspondante sera elle aussi à peine supérieure à 0 , ce qui ne saurait etre admis, car nous devons non seulement avoir $\pi / 2>0_{\mathrm{s}}>0$, mais encore

$$
0_{\mathrm{s}}>\varepsilon=\operatorname{Arctg} \frac{x_{0}}{y_{0}-a} .
$$

Cette inégalité ne pouvant être aisément introduite pour compléter les systèmes d'équations présentés aux $\$ \$ 2.1 .1$ et 2.1.2, il y aura donc lieu, lorsque $0_{\mathrm{s}}$ et $a$ auront été déterminés, de vérifier que l'inégalité :

$$
\theta_{\mathrm{S}}>\operatorname{Arctg}\left(x_{0} / y_{0}-a\right)
$$

est bien respectée.

\section{2-Cas où le cercle passe par $A$ et $B$ :}

En faisant passer le cercle par les points $A$ et $B$, nous nous imposons une condition supplémentaire et nous pouvons tout au plus espérer trouver des valeurs particulières de $y_{0}$ telles que toutes les conditions puissent être satisfaites. Nous reprendrons, dans le même ordre, les trois cas possibles selon les valeurs relatives de $y_{0}$ et de $a$.

2.2.1 - Cas où $y_{0}>a$ (cas de la figure 13): avec les notations de la figure 13 , nous avons les relations suivantes:

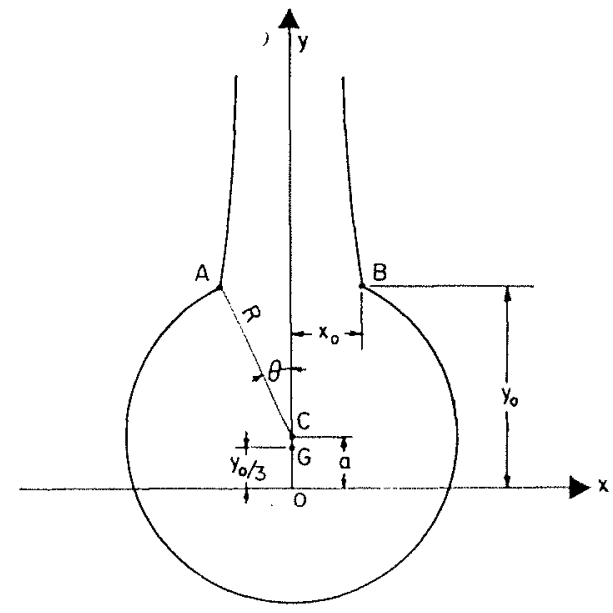

Fig. 13 


$$
\begin{gathered}
a+\mathrm{R} \cos \theta=y_{0} \\
\mathrm{R} \sin \theta=x_{0}=\sqrt{\mathrm{A} / y_{0}}
\end{gathered}
$$

$\mathrm{R}^{2}[(\pi-\theta)+\sin \theta \cos \theta]$

$$
\begin{array}{r}
=\mathrm{S}=4 \mathrm{~A} / x_{0}=4 \sqrt{\mathrm{A} y_{0}} \\
a-2 \mathrm{R}^{3} \sin ^{3} \theta / 3 \mathrm{~S}=y_{0} / 3
\end{array}
$$

dont la combinaison permet d'établir tout d'abord la relation intermédiaire

$$
x_{0}{ }^{3}=\mathrm{A}\left[\frac{\sqrt{9+4 \operatorname{tg}^{2} \theta}-3}{\operatorname{tg} \theta}\right]
$$

et enfin l'équation unique

$$
(\pi-\theta)=\sin 2 \theta\left[1+\sqrt{9 / 4+\operatorname{tg}^{2} \theta}\right]
$$

La solution $\theta_{\mathrm{S}}$ de l'équation (12) correspond donc à l'intersection des courbes $y_{1}(\theta)$ et $y_{2}(\theta)$ d'équations :

$$
\begin{aligned}
& y_{1}(\theta)=\pi-\theta \\
& y_{2}(\theta)=\sin 2 \theta\left[1+\sqrt{9 / 4+\operatorname{tg}^{2} \theta}\right]
\end{aligned}
$$

Une construction rapide donne l'allure de la courbe figure 14; par approximations successives l'on trouve rapidement la valeur unique $\theta_{\mathrm{s}}=39,56 \mathrm{gr}$, solution de (12).

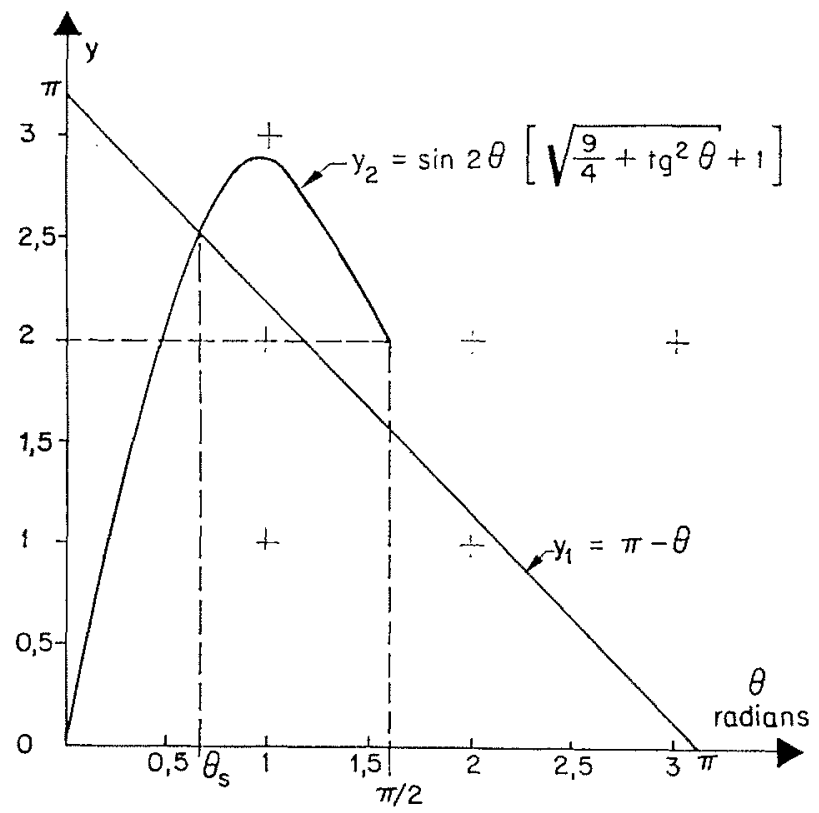

FIG. 14

Dans ces conditions, en portant dans (11) nous trouvons :

$x_{0}=0,76795 \sqrt[3]{\mathrm{A}}$

$\left(\right.$ ou $\left.\log x_{0}=(\log \mathrm{A}) / 3+\overline{1}_{,}, 88533\right)$ cette valeur de $x_{0}$ et Ia valeur $\theta_{\mathrm{S}}$ permettent de calculer $R$ d'après (8);

$$
\left.\begin{array}{rl}
R= & 1,3191 \sqrt[3]{A} \\
\quad(\text { ou } \log R
\end{array}=(\log A) / 3+0,12027\right)
$$

Enfin, compte tenu de (7) et des valeurs trouvées pour $x_{0}$ et $R$, il vient:

$a=0,62314 \sqrt[3]{\mathrm{A}}$

$$
\text { (ou } \log a=(\log \mathrm{A}) / 3+\overline{1}, 79459)
$$

A chaque valeur de A correspond donc une seule solution, définie entièrement par les relations (13), (14) et (15).

2.2.2 - Cas où $y_{0}<a$ (cas de la figure 15) : avec les notations de la figure 15 , nous avons les relations suivantes :

$$
\begin{gathered}
a-\mathrm{R} \cos \theta=y_{0} \\
\mathrm{R} \sin \theta=x_{0}
\end{gathered}
$$

$\mathrm{R}^{2}[\theta-\sin \theta \cos \theta]=\mathrm{S}=4 \sqrt{\overline{\mathrm{A}} \overline{y_{0}}}=4 \mathrm{~A} / x_{0}\left(9^{\prime}\right)$

$$
a-2 \mathrm{R}^{3} \sin ^{3} \theta / 3 \mathrm{~S}=y_{0} / 3
$$

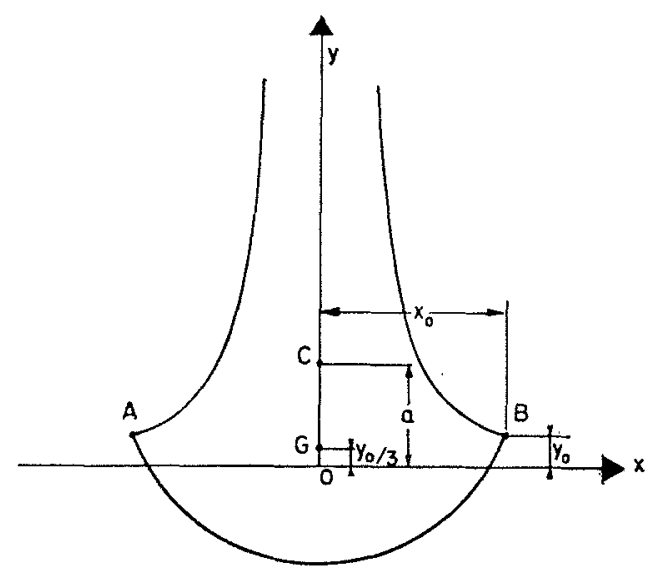

Fig. 15

Par un raisonnement en tous points comparable à celui développé pour le cas de figure 13, l'on trouve :

\section{et finalement}

$$
x_{0}^{3}=\mathrm{A}\left[\frac{\sqrt{9+4 \operatorname{tg}^{2} \theta}+3}{\operatorname{tg} \theta}\right]
$$

$$
\theta=\sin 2 \theta\left[\sqrt{9 / 4+\operatorname{tg}^{2} \theta}-1\right]
$$

La solution sera donc donnée par l'intersection des courbes $y_{3}(\theta)$ et $y_{4}(\theta)$ d'équations

$$
\begin{aligned}
& y_{3}(\theta)=\theta \\
& y_{4}(\theta)=\sin 2 \theta\left[\sqrt{9 / 4+\operatorname{tg}^{2} \theta}-1\right]
\end{aligned}
$$


Une étude très rapide dont nous ne donnons pas le détail permet de conclure que l'on a toujours (fig. 16) ;

$$
\sin 2 \theta\left[\sqrt{9 / 4+\operatorname{tg}^{2} \theta}-1\right] \geqslant 0
$$

et que l'égalité $\left(12^{\prime}\right)$ ne peut se vérifier que si $\theta=0$; le cas de figure 15 ne peut donc se réaliser que pour $\mathrm{R} \rightarrow \infty\left(\mathrm{R}=x_{0} / \sin \theta\right)$, le cercle se confondant alors avec l'axe $\overrightarrow{O x}$ et la courbe $y=\mathrm{A} / x^{2}$ étant alors utilisée dans sa totalité.

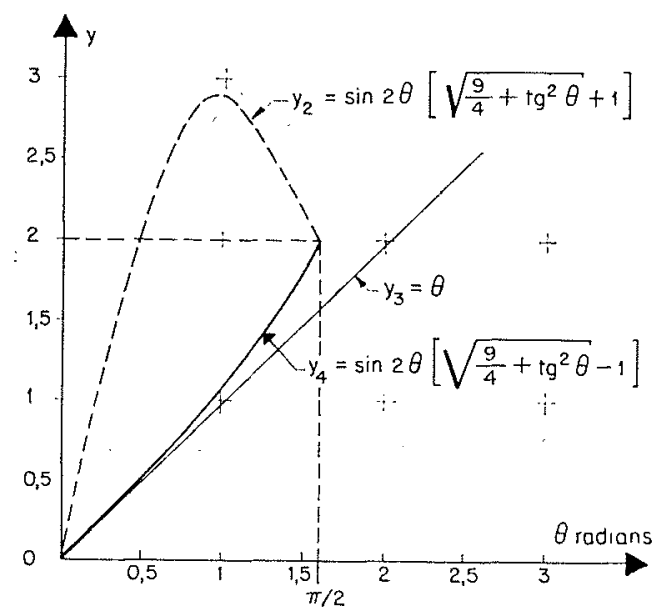

FiG. 16

2.2.3 - Cas où $y_{0}=a:$ le système d'équation devient :

$$
\begin{gathered}
a=y_{o} \\
\mathrm{R}=x_{0} \\
\mathrm{~S}=\pi \mathrm{R}^{2} / 2=4 \sqrt{\mathrm{Ay_{0 }}}=4 \mathrm{~A} / x_{0} \\
a-2 \mathrm{R}^{3} / 3 \mathrm{~S}=y_{0} / 3
\end{gathered}
$$

En faisant $a=y_{0}$ d'après $\left(7^{\prime \prime}\right)$ et $\mathrm{S}=\pi \mathrm{R}^{2} / 2$ dans $\left(10^{\prime \prime}\right)$, l'on trouve :

$$
\mathrm{R}^{2}=\pi^{2} \mathrm{~A}^{2} / 4 x_{0}{ }^{4}
$$

Or d'après $\left(9^{\prime \prime}\right)$, nous avons

$$
\mathrm{R}^{2}=8 \mathrm{~A} / \pi x_{0}
$$

En égalant ces deux valeurs de $R$ nous déterminons :

$$
x_{0}=\pi \sqrt[3]{\mathrm{A} / 32}
$$

Au contraire, en égalant la valeur déjà utilisée :

$$
\mathrm{R}^{2}=\pi^{2} \mathrm{~A}^{2} / 4 x_{0}^{4},
$$

et celle déduite de $\left(8^{\prime \prime}\right)$ soit $\mathrm{R}^{2}=x_{0}{ }^{2}$, l'on trouve $x_{0}=\sqrt[3]{\pi \mathrm{A} / 2}$.

Ces deux valeurs de $x_{0}$ étant différentes, nous en concluons que le cas $« y_{0}=a »$ est impossible.

2.2.4-Conclusions : en résumé, lorsque nous imposons au cercle de passer par les points A et B, il n'existe qu'une seule solution pour chaque valeur du paramètre $A$; c'est alors le cas de la figure 13 qui est valable, soit $y_{0}>a$.

Notons ici que, si nous considérons la famille des courbes d'équation $y=\mathrm{A} / x^{2}$ et si nous donnons au paramètre $A$ les valeurs $A_{0}, A_{1}, A_{2} \ldots$ $A_{p} \ldots$, les points $A$ et $B$ correspondants s'alignent sur deux droites passant par l'origine et de pente $m= \pm 1,69565 / 0,76795$, puisque ces points sont tels que nous ayons :

$$
\left\{\begin{array}{l}
x_{0}= \pm 0,76795 \sqrt[3]{\mathrm{A}} \\
y_{0}=+1,69565 \sqrt[3]{\mathrm{A}}
\end{array}\right.
$$

Par ailleurs, nous pouvons remarquer que les tangentes en $\mathrm{B}$ à la courbe $y=\mathrm{A} / x^{2}$ d'une part, et au cercle d'autre part ont respectivement les pentes suivantes :

a) pour la courbe $: y=\mathrm{A} / x^{2}$ :

$$
y_{0}^{\prime}=-2 \mathrm{~A} / x_{0}{ }^{3}=-2 /(0,76795)^{3}=-4,4162
$$

b) pour le cercle :

$$
\begin{aligned}
& m_{0}=\operatorname{tg}(\pi / 2-\alpha)=x_{0}{ }^{3} /\left(\mathrm{A}-a x_{0}{ }^{2}\right) \\
& =(0,76795)^{3} /\left[1-(0,63314)(0,76795)^{2}\right]=0,7160
\end{aligned}
$$

Nous en déduisons de plus :

$$
\alpha \# 60,4 \mathrm{gr} \quad \text { et } \beta \# 85,8 \mathrm{gr}
$$

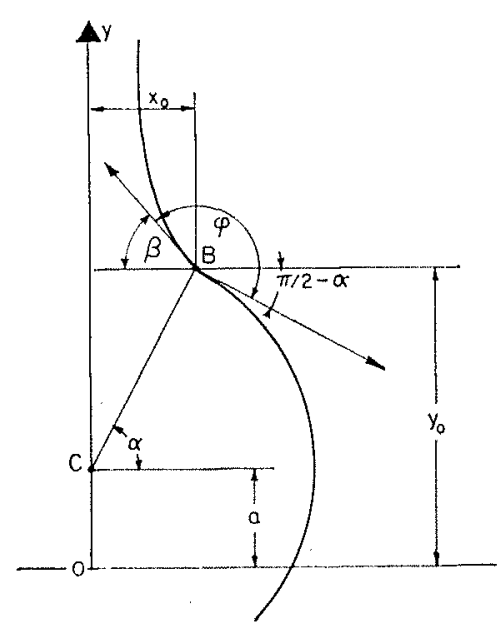

FIG. 17 
Nous pouvons donc dire que la solution trouvée présente les caractéristiques remarquables suivantes :

- Si $A_{0}$ est une valeur donnée du paramètre $A, B_{0}$ étant le point de rencontre du cercle avec la courbe $y=A / x^{2}, C_{0}$ étant le centre du cercle correspondant et si nous considérons d'autres valeurs $\mathrm{A}_{1}, \mathrm{~A}_{2}, \ldots A_{\mathrm{p}} \ldots$, , du paramètre, auxquelles correspondent autant de points $\left(B_{1}, C_{1}\right)$, $\left(B_{2}, C_{2}\right) \ldots\left(B_{1}, C_{p}\right) \ldots$, nous pourons dire que les triangles $\mathrm{OB}_{0} \mathrm{C}_{0}, \mathrm{OB}_{1} \mathrm{C}_{1}, \ldots \mathrm{OB}_{p} \mathrm{C}_{p} \ldots$ sont semblables.

- Aux points $B_{0}, B_{1}, B_{2} \ldots B_{p} \ldots$, (ei aux points $A_{0}, A_{1}, A_{2} \ldots A_{p} \ldots$ quj leur sont associés), les tangentes au cercle d'une part, et à la courbe $y=\mathrm{A} / x^{2}$ d'autre part, font entre elles un angle constant, tel que

$$
\varphi \# 153,7 \mathrm{gr} \text {. }
$$

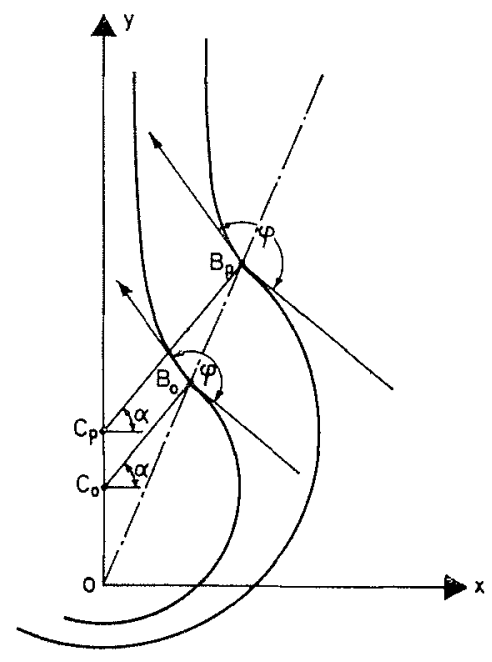

FIG. 18

\section{$3^{\circ}$ Chorx de la section Éourvalente:}

Les surfaces simples que nous avons seules ćtudiées, à savoir le cercle el le rectangle, reprćsentent des solutions du problème de la section équivalente dont nous avons donné au fur et à mesure les conditions d'existence, et en quelque sorte les degrés de liberté. Bien que ces aires soient théoriquement équivalentes, du moins si nous considérons des domaines où les solutions correspondantes coexistent, nous pensons que le remplacement d'un rectangle par un cercle doit avoir un heureux effet sur le coefficient de débit de l'ensemble; dans cet ordre d'idées, c'est le cas traité aux $\$ \$ 2.2 .1$ et 2.2.4 qui parait devoir posséder cet avantage au plus haut point, un inconvénient résultant toutefois de l'unicité de la solution pour chaque valeur du paramètre A. Nous regrettons ici tout particulièrement qu'il ne nous ait pas été possible de procéder à des essais comparatifs des deux types de sections équivalentes.

\section{Les déversoirs en " tour Eiffel»: existence d'un profil type}

Alors que nous avons démontré l'équivalence thcorique des deux définitions du profil, soit

$$
x^{2} y=3 k^{2} / 64 g \beta^{2} \theta^{2} \cong k k^{2} / g \pi^{2} \theta^{2},
$$

il y a lieu de considérer qu'en pratique il n'en est pas ainsi, car le coefficient $\beta$ n'est plus égal à $\pi \sqrt{3} / 4 \sqrt{2}$, mais est inférieur à cette valeur, en raison du remplacement de la partie asymptotique par une section équivalente.

Dans ces conditions, nous ne retenons que l'expression modifiée de P. Morin, soit

$$
x^{2} y=3 k^{2 / 64} g \beta^{2} \theta^{2}
$$

et nous remarquons immédiatement que cette relation peut s'écrire :

$$
x=\sqrt{\frac{3}{64 g}} \cdot \sqrt{\frac{1}{y}} \cdot \frac{k}{\beta \theta} ;
$$

le rapport $(k / \beta \theta)$ joue donc le rôle d'un paramètre caractéristique et l'ensemble des profils $x(y)$ se déduit de l'un d'eux grâce à une simple affinité par rapport à $\overrightarrow{\mathrm{O}} \overrightarrow{\mathrm{y}}$.

Finalement, si nous appelons $C_{100}$ le «profil type », tel que la valeur correspondante du paramètre soit $k / \beta \theta=100$, et $(C)_{p}$ le profil tel que nous ayons $k / \beta \theta=p$, nous aurons, pour une même valeur de $y$, la relation :

$$
x_{j} / x_{100}=p / 100 \text {. }
$$

Le tableau I donne les valeurs de $\log \left(x_{100}\right)$ pour toutes les valeurs de $y$ allant de 2 en $2 \mathrm{~mm}$ depuis $2 \mathrm{~cm}$ jusqu'à $30 \mathrm{~cm}$. 
Tableau I

\begin{tabular}{|c|c|}
\hline$y^{\mathrm{cm}}$ & $\log \left(x_{100}\right)$ \\
\hline 2,0 & $\overline{1,68912}$ \\
\hline 2,2 & 66842 \\
\hline 2,4 & 64953 \\
\hline 2,6 & 63215 \\
\hline 2,8 & 61605 \\
\hline 3,0 & $\overline{1,60} 107$ \\
\hline 3,2 & 58706 \\
\hline 3,4 & 57389 \\
\hline 3,6 & 56148 \\
\hline 3,8 & 54974 \\
\hline 4,0 & $\overline{1,53860}$ \\
\hline 4,2 & 52801 \\
\hline 4,4 & 51791 \\
\hline 4,6 & 50825 \\
\hline 4,8 & 49901 \\
\hline 5,0 & $\overline{1,49015}$ \\
\hline 5,2 & 48163 \\
\hline 5,4 & 47344 \\
\hline 5,6 & 46554 \\
\hline 5,8 & 45792 \\
\hline 6,0 & $\overline{1,45056}$ \\
\hline 6,2 & 44344 \\
\hline 6,4 & 43654 \\
\hline 6,6 & 42986 \\
\hline 6,8 & 42338 \\
\hline 7,0 & $\overline{1,41708}$ \\
\hline 7,2 & 41097 \\
\hline 7,4 & 40502 \\
\hline 7,6 & 39923 \\
\hline 7,8 & 39359 \\
\hline 8,0 & 1,38809 \\
\hline 8,2 & 38273 \\
\hline 8,4 & 37749 \\
\hline 8,6 & 37238 \\
\hline 8,8 & 36739 \\
\hline 9,0 & 1,36251 \\
\hline 9,2 & 35774 \\
\hline 9,4 & 35307 \\
\hline 9,6 & 34850 \\
\hline 9,8 & 34402 \\
\hline 10,0 & $\overline{1,33963}$ \\
\hline 10,2 & 33533 \\
\hline 10,4 & 33112 \\
\hline 10,6 & 32698 \\
\hline 10,8 & 32292 \\
\hline 11.0 & $\overline{1,31894}$ \\
\hline 11,2 & 31502 \\
\hline
\end{tabular}

\begin{tabular}{|c|c|}
\hline$y^{\mathrm{rm}}$ & $\log \left(x_{1 \oplus \infty}\right)$ \\
\hline $\begin{array}{l}11,4 \\
11,6 \\
11,8\end{array}$ & $\begin{array}{r}1,31118 \\
30740 \\
30369\end{array}$ \\
\hline 12,0 & $\overline{1,30004}$ \\
\hline 12,2 & 29645 \\
\hline 12,4 & 29292 \\
\hline 12,6 & 28945 \\
\hline 12,8 & 28603 \\
\hline 13,0 & $\overline{1,28} 266$ \\
\hline 13,2 & 27935 \\
\hline 13,4 & 27608 \\
\hline 13,6 & 27286 \\
\hline 13,8 & 26969 \\
\hline 14,0 & $\overline{1}, 26657$ \\
\hline 14,2 & 26349 \\
\hline 14,4 & 26045 \\
\hline 14,6 & 25746 \\
\hline 14,8 & 25450 \\
\hline 15,0 & $\overline{1,25} 159$ \\
\hline 15,2 & 24871 \\
\hline 15,4 & 24587 \\
\hline 15,6 & 24307 \\
\hline 15,8 & 24030 \\
\hline 16,0 & 1,23757 \\
\hline 10,2 & 23487 \\
\hline 16,4 & 23221 \\
\hline 16,6 & 22958 \\
\hline 16,8 & 22698 \\
\hline 17,0 & $\overline{1,22} 441$ \\
\hline 17,2 & 22187 \\
\hline 17,4 & 21936 \\
\hline 17,6 & 21688 \\
\hline 17,8 & 21442 \\
\hline 18,0 & $\overline{1}, 21200$ \\
\hline 18,2 & 20960 \\
\hline 18,4 & 20722 \\
\hline 18,6 & 20488 \\
\hline 18,8 & 20255 \\
\hline 19,0 & $\overline{\mathbf{1}, 20} 026$ \\
\hline 19,2 & 19798 \\
\hline 19,4 & 19573 \\
\hline 19,6 & 19350 \\
\hline 19,8 & 19130 \\
\hline 20,0 & $\overline{1,18912}$ \\
\hline 20,2 & 18696 \\
\hline 20,4 & 18482 \\
\hline 20,6 & 18270 \\
\hline
\end{tabular}

\begin{tabular}{|c|c|}
\hline$y^{\mathrm{cm}}$ & $\log \left(x_{101}\right)$ \\
\hline 20,8 & 18060 \\
\hline 21,0 & 1,17852 \\
\hline 21,2 & 17646 \\
\hline 21,4 & 17443 \\
\hline 21,6 & 17241 \\
\hline 21,8 & 17040 \\
\hline 22,0 & 116842 \\
\hline 22,2 & 16646 \\
\hline 22,4 & 16451 \\
\hline 22,6 & 16258 \\
\hline 22,8 & 16067 \\
\hline 23,0 & 1,15877 \\
\hline 23,2 & 15689 \\
\hline 23,4 & 15502 \\
\hline 23,6 & 15318 \\
\hline 23,8 & 15134 \\
\hline 24,0 & 1,14953 \\
\hline 24,2 & 14772 \\
\hline 24,4 & 14594 \\
\hline 24,6 & 14416 \\
\hline 24,8 & 14241 \\
\hline 25,0 & 1,14066 \\
\hline 25,2 & 13893 \\
\hline 25,4 & 13722 \\
\hline 25,6 & 13551 \\
\hline 25,8 & 13382 \\
\hline 26,0 & 1,13215 \\
\hline 26,2 & 13048 \\
\hline 26,4 & 12883 \\
\hline 26,6 & 12719 \\
\hline 26,8 & 12557 \\
\hline 27,0 & 1,12395 \\
\hline 27,2 & 12235 \\
\hline 27,4 & 12076 \\
\hline 27,6 & 11918 \\
\hline 27,8 & 11761 \\
\hline 28,0 & 1,11605 \\
\hline 28,2 & 11451 \\
\hline 28,4 & 11297 \\
\hline 28,6 & 11145 \\
\hline 28,8 & 10994 \\
\hline 29,0 & 1,10843 \\
\hline 29,2 & 10694 \\
\hline 29,4 & 10546 \\
\hline 29,6 & 10399 \\
\hline 29,8 & 10252 \\
\hline 30,0 & 1,10107 \\
\hline
\end{tabular}




\section{II. - ETUDES EXPERIMENTALES : exposé des résultats, discussion}

Après avoir rappelé sommairement, faute d'indications plus complètes, les résultats obtenus à propos des Sutro-Weirs, nous insistons particulièrement sur les déversoirs en Tour Eiffel; après un rappel des résultats obtenus d'abord par P.A. Roubinet, puis par P. Morin, nous exposons en détail les essais que nous avons pu faire, et en comparons les résultats aux précédents. Dans ce domaine expérimental nous ne retrouvons malheureusement pas le nom de L. Hugues, cet auteur n'ayant pas publié de résultats d'essais, à notre connaissance du moins.

1 - Résultats Relatifs aux Sutro-Weirs : nous n'insisterons pas sur les essais relatifs à de tels déversoirs, notre expérience personnelle ayant été essentiellement celle des déversoirs en «Tour Eiffel », mais nous pensons qu'il est intéressant d'indiquer rapidement les résultats concernant les valeurs du coefficient de débit $\theta$, et ce afin de les comparer à celles que nous avons trouvées après P. A. Roubinet et P. Morin.

Dans ces conditions, nous indiquerons simplement qu'il ressort des essais de Di Ricco une valeur moyenne de 0,6168 pour le coefficient de débit $\theta$, cette valeur variant par contre d'après les auteurs américains de 0,605 à 0,625 en fonction d'une part de $h$ (charge) et d'autre part de $b$ (largeur de base de l'échancrure). Il importe enfin de préciser que ces dernières valeurs correspondent à un demi-profil et doivent être augimentées de $1 \%$ pour le déversoir symétrique.

$2^{\circ}$ RÉsultats DE P.A. Roubinet : cet auteur n'ayant publié que la note déjà citée [13], c'est de cette publication que nous tirons les éléments

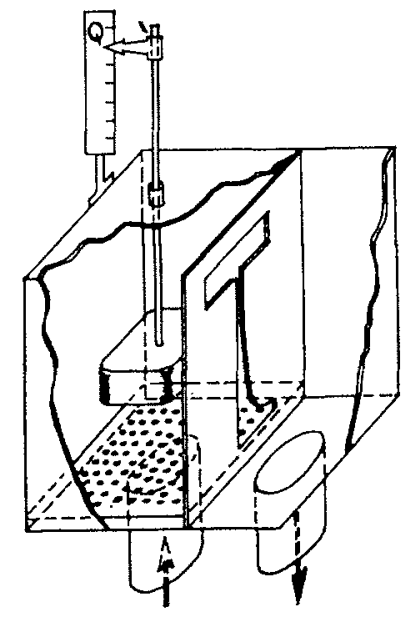

FIG. 19 suivants : «le déversoir était taillé dans une plaque de laiton de $3 \mathrm{~mm}$ d'épaisseur par un ajusteur habile, mais celui-ci ne disposait d'aucun outillage spécial de traçage ou de vérification. Le déversoir ainsi construit a été fixé sur la cloison d'une petite bâche cubique en tôle de $20 \mathrm{~cm}$ de côté» suivant figure 19 .

Nous préciserons qu'il s'agissait d'un demiprofil, caractérisé par la valeur du parametre $\alpha=2$, et se terminant à la partie inférieure par un rectangle de $1 \mathrm{~mm}$ de haut $\left(=1 / 100^{\circ}\right.$ de la charge maximale, soit $10 \mathrm{~cm}$ ) et de $32 \mathrm{~mm}$ de largeur $(3,2 \# 1 / \sqrt{0,1})$.

Nous pouvons remarquer à ce propos que le demi-profil constitue une solution théoriquement indistincte de celle du profil entier; en pratique toutefois l'on constate que le profil symétrique présente un-coefficient de débit $\theta$ légèrement supérieur.

Les essais faits par l'auteur sur cette seule plaque lui ont permis de calculer une valeur du coefficient de contraction : $m^{\prime}=0,54$. Or nous avons déjà démontré (cf. "études théoriques» - travaux de P. Morin) que Ie coefficient $m^{\prime}$ du profil P.A. Roubinet était lié au coefficient $m$ du profil de $\mathrm{P}$. Morin par Ia relation :

$$
m^{\prime}=m \sqrt{2 / 3}
$$

qui nous permet de conclure que le coefficient de contraction réel n'est pas de 0,54 , mais est égal à : $0,54 \sqrt{3 / 2}$, soit voisin de 0,66 ; si nous considérons enfin que cette valeur a été obtenue dans le cas d'un demi-prófil, nous pouvons penser qu'en fait le coefficient de contraction $m$, ou plus exactement le coefficient de débit avec lequel il a été confondu, doit être de l'ordre de 0,67, dans le cas d'un profil symétrique.

Des conditions expérimentales, nous retiendrons surtout que l'essai fut réalisé dans un bac de dimensions très modestes et que le compartiment amont était alimenté par le fond; nous aurons à revenir sur cette remarque dans ce qui suit.

$3^{\circ}$ Résultats de P. Morin : dans la note déjà citée [9], l'auteur décrit les expériences qu'il a réalisées avec deux plaques-déversoirs dont le paramètre $A$ avait les valeurs suivantes :

$$
\begin{aligned}
& \text { plaque «A»: } A_{1}=1,475 \\
& \text { plaque } \ll B »: A_{2}=0,288,
\end{aligned}
$$

la plaque $A$ ayant un débit capable de 6 à $125 \mathrm{l} / \mathrm{mn}$ et la plaque $\mathrm{B}$ un débit capable de 3 à $30 \mathrm{l} / \mathrm{mn}$. 
Ces plaques rectangulaires de $25 \times 30 \mathrm{~cm}$ étaient en duralumin de $2 \mathrm{~mm}$ d'épaisseur; elles étaient fixées, pour les essais, sur une cuve de jauge à trois compartiments alimentés par un écoulement réglable par une vanne (fig. 20).

Dans le compartiment A débouchait la conduite d'alimentation, les compartiments B et C étant alors tous deux alimentés par le fond, le premier portant sur sa face avant la plaque à essayer (5) grâce à un système de joint hydraulique, et le second étant destiné aux mesures de niveau.

Les mesures de débit étaient faites par empotement dans un seau de 21 litres, avec une erreur de $2 / 10^{\text {es }}$ de seconde et les mesures de niveau au millimètre près.

Les résultats des essais présentés sous la forme d'une courbe $\mathrm{Q}=f(y)$ mettent en évidence l'alignement des points sur deux droites $\mathrm{Q}=k y$, telles que l'on ait :

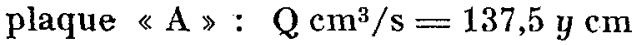

$$
\begin{aligned}
& \text { plaque « } \mathrm{B} »: \mathrm{Q} \mathrm{cm}^{3} / \mathrm{s}=56,7 \mathrm{~g} \mathrm{~cm} \text {, }
\end{aligned}
$$

et si l'on considère que nous avons posé :

$$
\mathrm{A}=3 k^{2} / 64 m^{2} g
$$

il est facile de calculer les valeurs suivantes du coefficient de contraction $m$

$$
\begin{aligned}
& \text { plaque } \ll \mathbf{A} »: \quad m \# 0,78 \\
& \text { plaque } \ll \mathbf{B} »: m \# 0,73,
\end{aligned}
$$

ce qui amène l'auteur à admettre une valeur moyenne : $m$ \# 0,75.

Dans une discussion intéressante sur la précision de ses mesures, l'auteur montre que l'erreur absolue faite sur le débit est de I'ordre du litre/minute au maximum. Cette valeur a été dépassée pour une seule mesure, qui peut donc être considérée comme entachée d'erreurs excessives; pour les faibles valeurs de la charge les erreurs observées (écart entre les valeurs réelle d'une part et calculée d'après $\mathrm{Q}=k y$ d'autre part) sont bien de l'ordre de grandeur du litre/minute, alors qu'elles tendent dans l'ensemble à diminuer lorsque la charge augmente, et ce environ à partir de la moitié de la charge maximale.

$4^{\circ}$ DÉtall DE Nos RÉSUltats - nous avons fait construire trois plaques déversoirs, de paramètres :

$$
A_{1}=4,3004 \quad A_{2}=7,6452 \quad A_{3}=17,2016
$$

(5) On peut à ce propos remarquer sur le croquis correspondant que les deux profils ont été en fait découpés dans la même plaque et opposés par leurs sommets.

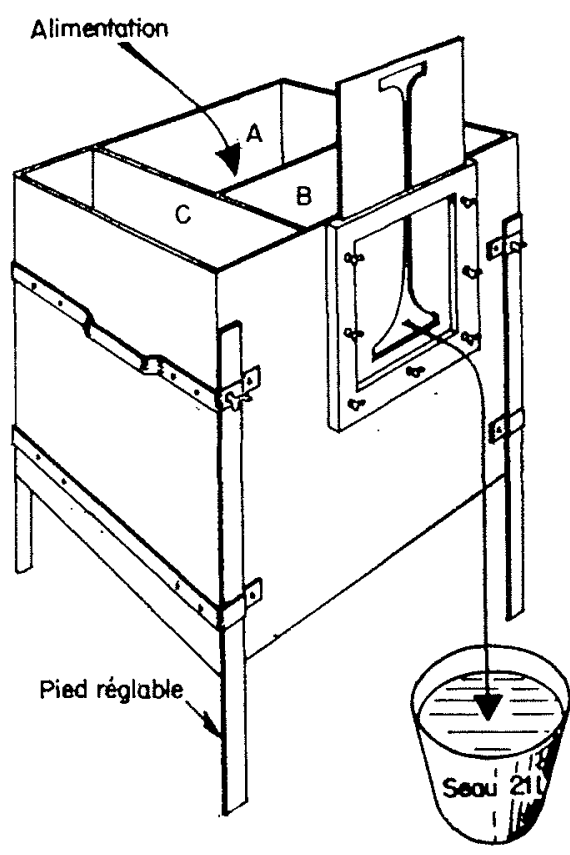

FIG. 20

(correspondant à des valeurs $k / \beta 0$, respectivement égales à 300,400 et 600 ) (cf. tableau II).

Les sections équivalentes ont été choisies rectangulaires. Pour les trois profils nous avons pris : $y_{0}=2 \mathrm{~cm}$, d'où la hauteur du rectangle : $h_{0}=4 y_{0} / 3=2,666 \mathrm{~cm}$; la largeur $l_{0}$ du rectangle diffère selon les profils puisque, rappelons-le, nous avons : $l_{0}=3 x_{0}=3 \sqrt{\mathrm{A} / y_{0}}$, et les différentes valeurs de $l_{0}$ sont respectivement : $\left(l_{0}\right)_{1}=4,4 \mathrm{~cm}, \quad\left(l_{0}\right)_{2}=5,865 \mathrm{~cm}, \quad\left(l_{0}\right)_{3}=8,8 \mathrm{~cm}$

Les profils ont été réalisés dans des plaques de laiton de 2 mm d'épaisseur, et de forme rectangulaire $(30 \times 40 \mathrm{~cm}$ pour les profils 1 et 3 , $30 \times 33 \mathrm{~cm}$ pour le profil 2$)$.

Les essais ont été faits au Laboratoire d'Hydraulique de l'Ecole Nationale du Génie Rural, dont nous n'avons utilisé que le canal vitré à inclinaison variable (pentes pouvant varier de $-3 \%$ à $+15 \%$ ).

La photographie 1 a donne une vue d'ensemble de ce canal d'une longueur nette de $8,50 \mathrm{~m}$, dont $6,75 \mathrm{~m}$ sont vitrés (en cinq panneaux), d'une largeur intérieure de $0,30 \mathrm{~m}$ et d'une profondeur de $0,50 \mathrm{~m}$. Les photographies $2 a, 2 b$ et $2 d$ nous montrent les feuillures à blocage d'écran permettant la fixation des diverses plaques dont on veut faire l'essai (6). Ce canal, pourvu de dispositifs d'uniformisation à l'amont (photographie $1 b$ ) et à l'aval (photos $1 c$ et $1 d$ ), peut être alimenté à un débit compris entre 0 et $300 \mathrm{l} / \mathrm{s}$.

(6) En raison des dimensions imposées par ailleurs a nos plaques, les essais ont été réalisés grâce à l'interposition d'un châssis en bois, visible sur les photos. 
Les plaques déversoirs étaient placées à l'extrćmité aval du troisième panneau vitré, soit 4,05 mètres après la sortie du dispositif amont d'uniformisation.

Des prises de pression établies dans le fond du canal (photo $1 c$ et vue détaillée de l'écoule- ment) permettent, soit de lire directement les niveaux sur un panneau de contrôle $P$, soit de renvoyer ces niveaux sur une pointe de mesure $M$ donnant le $1 / 10^{\circ}$ de $10 m$ (photo $1 b$ ).

Le débit peut être mesuré, soit par empotement dans un bac de 110 litres (photo $1 d$ ) pour

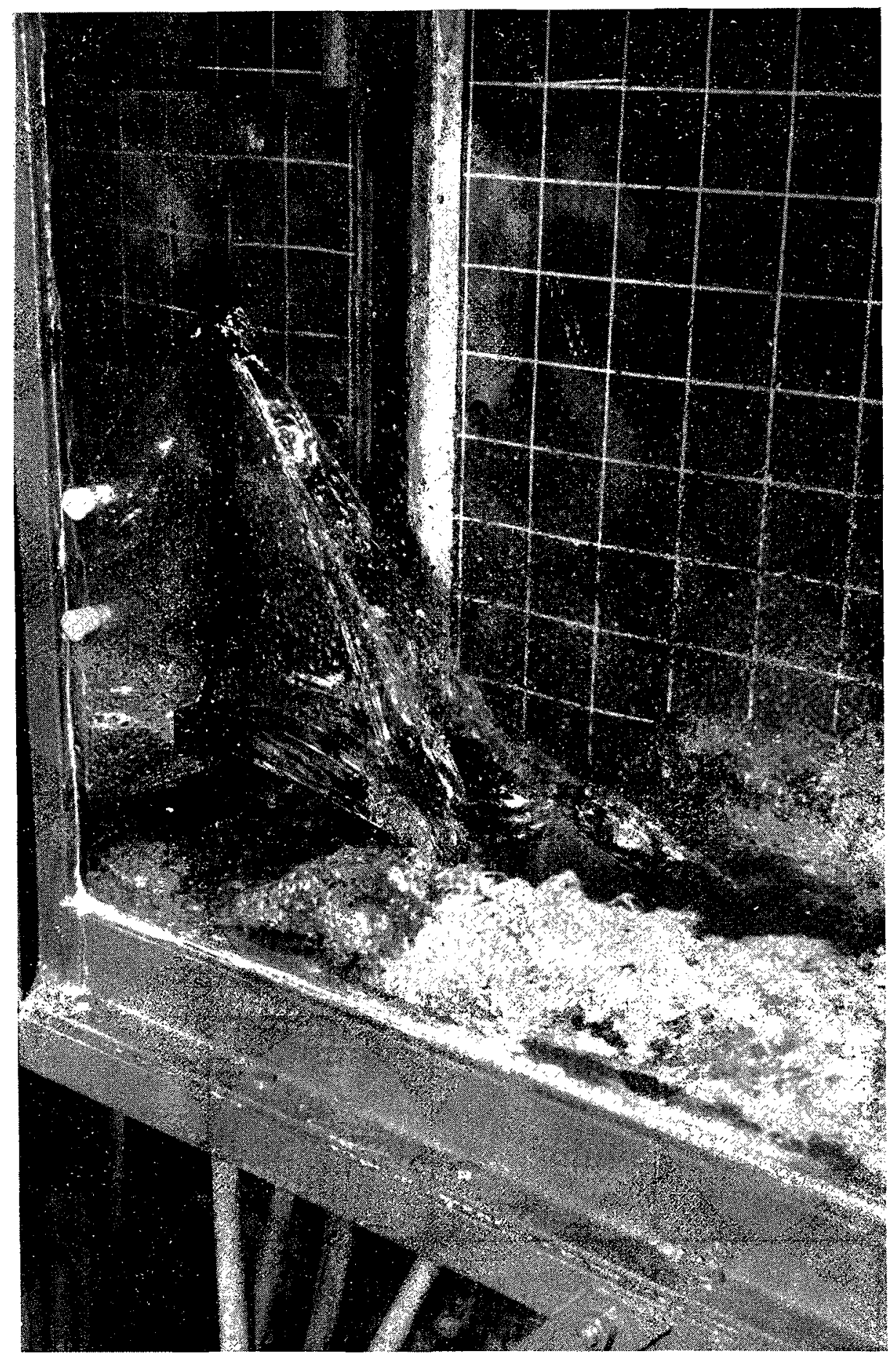

Vue détaillée de l'écoulement. 


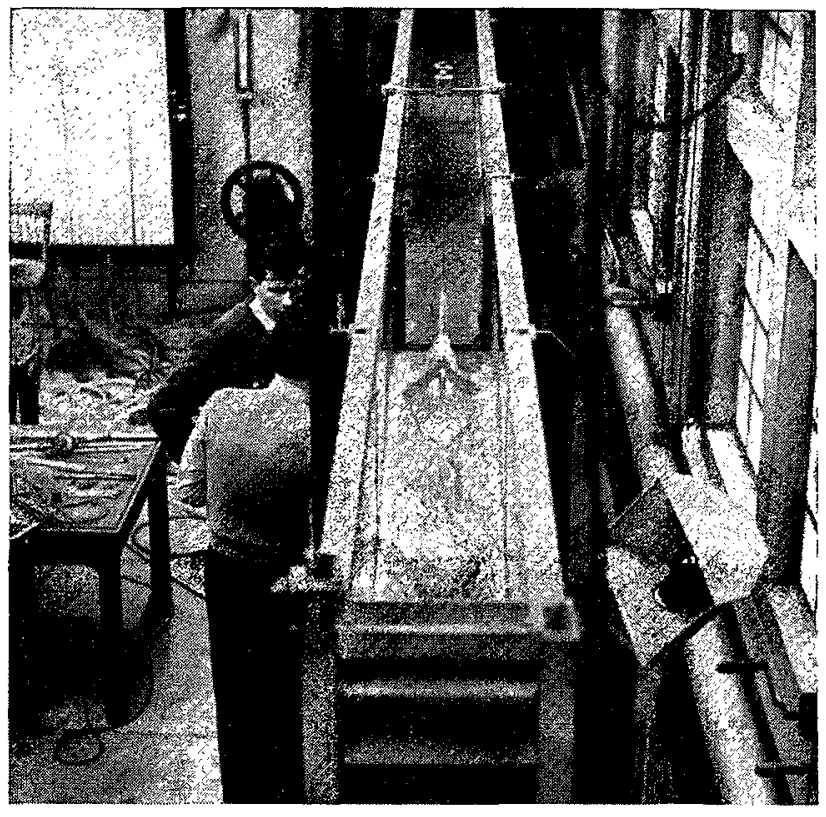

Pното 1 a Vue générale d'un essai.

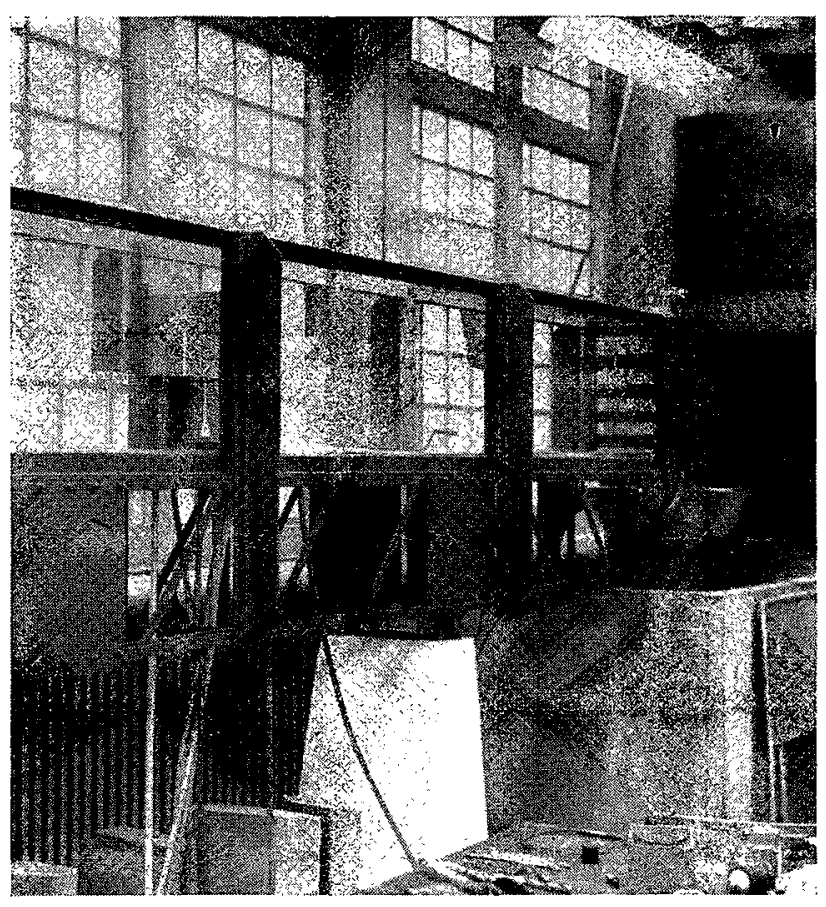

Рното $1 c$

Déversoir en place dans le canal

les petits débits $(<7 \mathrm{l} / \mathrm{s})$, soit par un Venturi placé sur la conduite d'alimentation du canal (et dont le dispositif de lecture $V$ est visible sur la photo $1 \mathrm{~b}$ ) pour les débits supérieurs à $7 \mathrm{l} / \mathrm{s}$. Nos trois séries d'essais ont été faites avec pente nulle du canal, lecture directe des niveaux

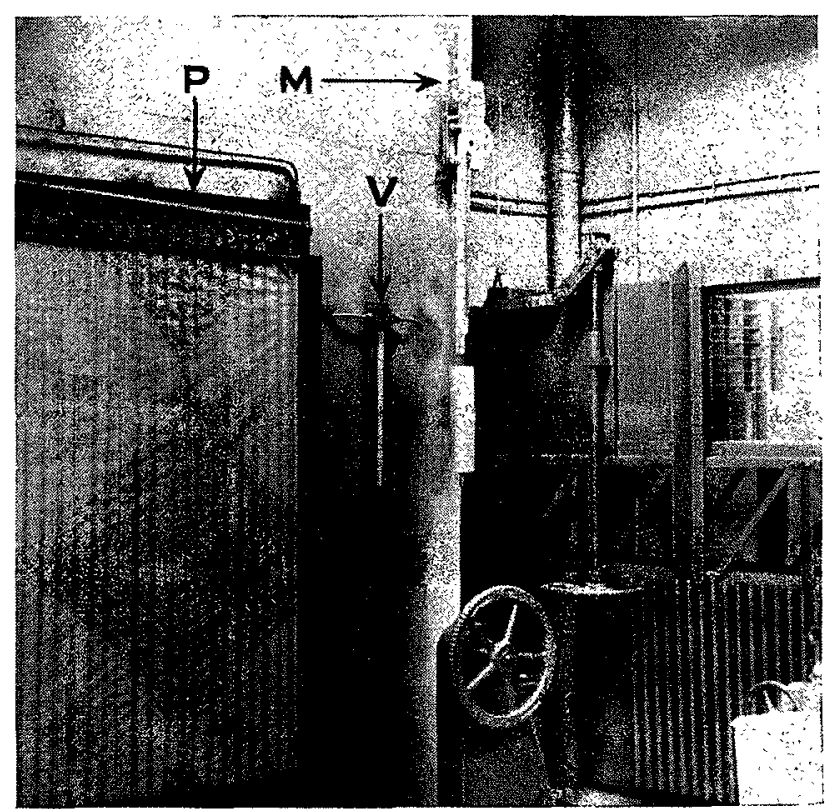

Pнore $1 \mathrm{~b}$

Pammeau de contrôle des niveaux.

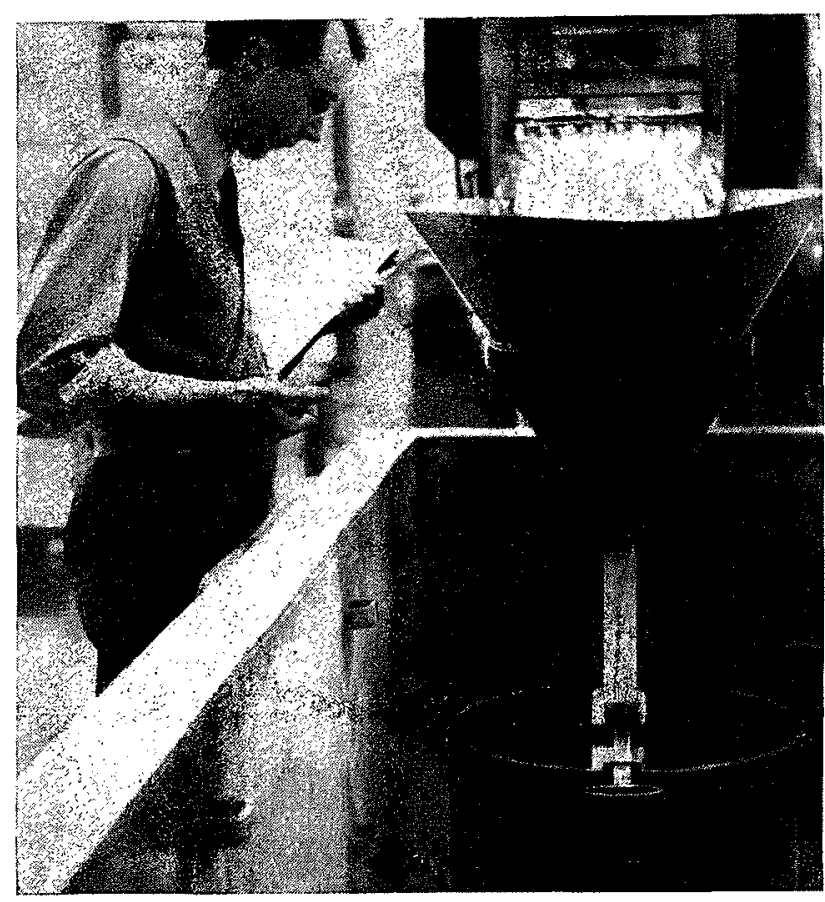

Photo 1 a

Dispositif de mesure du débit.

au $1 / 2 \mathrm{~mm}$ près, mesure des débits soit par empotement avec erreur de 0,2 litre (entre 100 et 110 litres) et $2 / 10^{\mathrm{es}}$ de seconde, soit par Venturi (avec emploi de la courbe d'étalonnage du Laboratoire).

La conduite des trois séries d'essais differe 


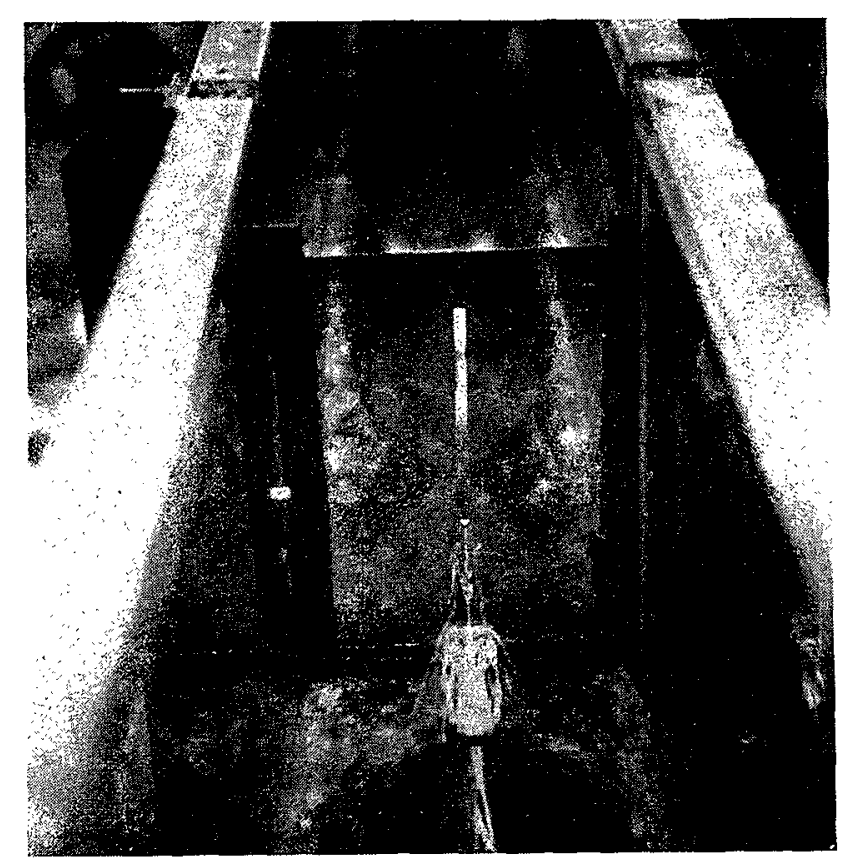

Рното 2 a

Vue aval de l'écoulement.

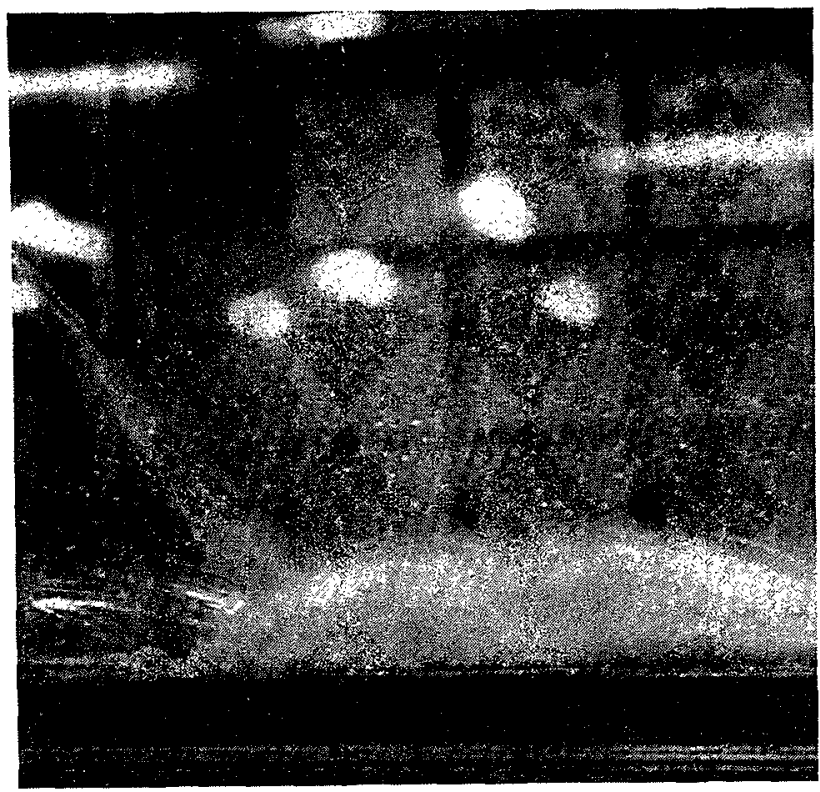

Рното $2 c$

Vue latérale de l'écoulement.

toutefois car, si le profil 2, expérimenté le premier, a été étudié à charge décroissante avec mesures couplées et correspondant à deux états très voisins, les profils, 1 et 3 ont été essayés à charges croissante et décroissante avec étalement des mesures. Ce deuxième mode opératoire

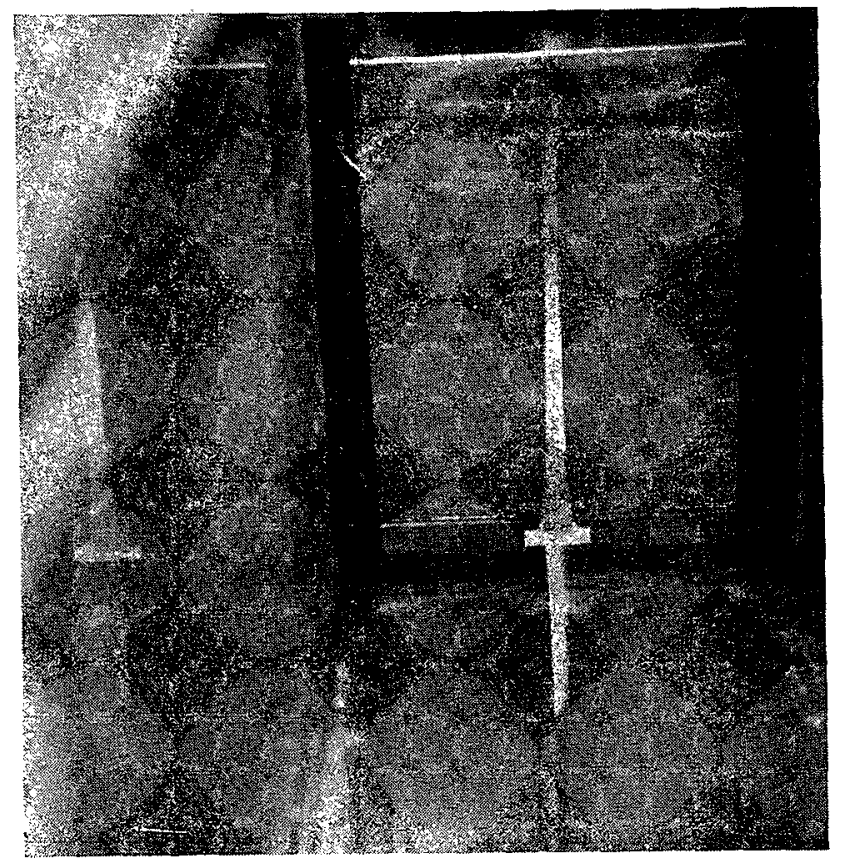

Рното $2 b$

Vue amont de l'écoulement.

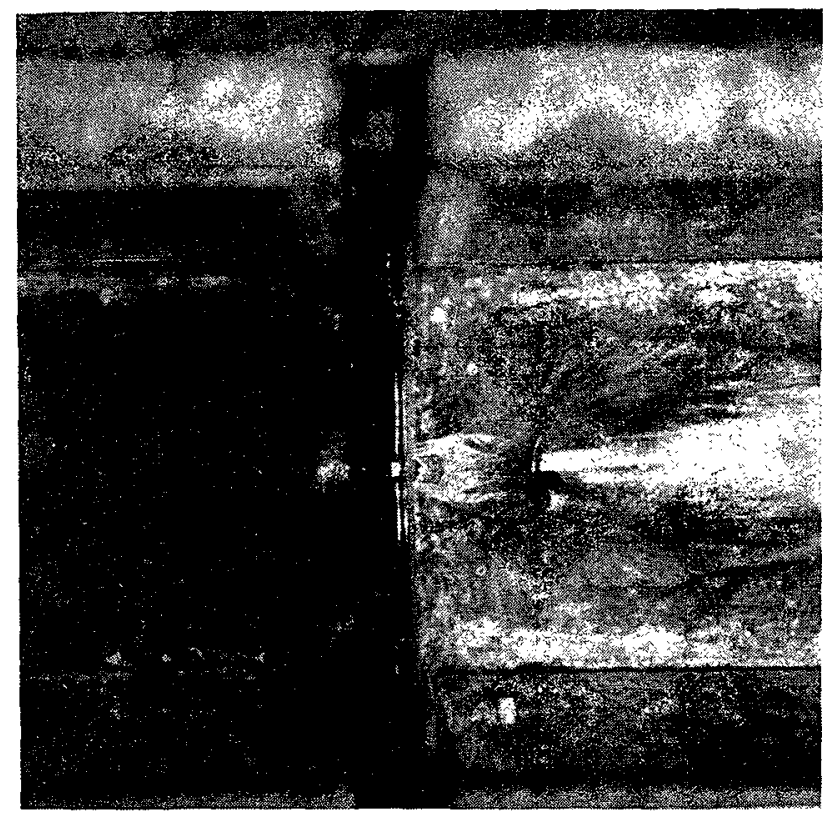

Pното $2 d$

Vue de dessus de l'écoulement.

nous a paru constituer une amélioration et pouvoir remédier à certains défauts apparus lors du premier essai, qu'il ne nous a malheureusement pas été possible de reprendre.

Les résultats des essais sont consignés dans le tableau III et le graphique de la figure 21 . 
TABLEAU II

\begin{tabular}{|c|c|c|c|}
\hline \multirow{2}{*}{$Y^{m m}$} & \multicolumn{3}{|c|}{$\mathrm{X}^{\mathrm{mm}}$} \\
\hline & Profil 1 & Profil 2 & Profil 3 \\
\hline 20 & 14,66 & 19,55 & 29,33 \\
\hline 22 & 13,98 & 18,64 & 27,96 \\
\hline 24 & 13,39 & 17,85 & 26,77 \\
\hline 26 & 12,86 & 17,15 & 25,72 \\
\hline 28 & 12,39 & 16,52 & 24,79 \\
\hline 30 & 11,97 & 15,96 & 23,95 \\
\hline 32 & 11,59 & 15,46 & 23,19 \\
\hline 34 & 11,25 & 14,99 & 22,49 \\
\hline 36 & 10,93 & 14,57 & 21,86 \\
\hline 38 & 10,64 & 14,18 & 21,28 \\
\hline 40 & 10,37 & 13,82 & 20,74 \\
\hline 42 & 10,12 & 13,49 & 20,24 \\
\hline 44 & 9,89 & 13,18 & 19,77 \\
\hline 46 & 9,67 & 12,89 & 19,34 \\
\hline 48 & 9,46 & 12,62 & 18,93 \\
\hline 50 & 9,27 & 12,37 & 18,55 \\
\hline 52 & 9,09 & 12,13 & 18,19 \\
\hline 54 & 8,92 & 11,90 & 17,85 \\
\hline 56 & 8,76 & 11,68 & 17,53 \\
\hline 58 & 8,61 & 11,48 & 17,22 \\
\hline 60 & 8,47 & 11,29 & 16,93 \\
\hline 64 & 8,20 & 10,93 & 16,39 \\
\hline 68 & 7,95 & 10,60 & 15,91 \\
\hline 72 & 7,73 & 10,30 & 15,46 \\
\hline 76 & 7,52 & 10,03 & 15,04 \\
\hline
\end{tabular}

\begin{tabular}{|c|c|c|c|}
\hline \multirow{2}{*}{$Y^{m+n}$} & \multicolumn{3}{|c|}{$X^{m m}$} \\
\hline & Profil 1 & Profil 2 & Profil 3 \\
\hline 80 & 7,33 & 9,78 & 14,66 \\
\hline 86 & 7,07 & 9,43 & 14,14 \\
\hline 92 & 6,84 & 9,12 & 13,67 \\
\hline 98 & 6,62 & 8,83 & 13,25 \\
\hline 104 & 6,43 & 8,57 & 12,86 \\
\hline 110 & 6,25 & 8,34 & 12.50 \\
\hline 120 & 5,99 & 7,98 & 11,97 \\
\hline 130 & 5,75 & 7.67 & 11,50 \\
\hline 140 & 5,54 & 7,39 & 11,08 \\
\hline 150 & 5,35 & 7,14 & 10,71 \\
\hline 160 & 5,18 & 6,91 & 10,37 \\
\hline 170 & 5,03 & 6,71 & 10,$06 ;$ \\
\hline 180 & 4,89 & 6,52 & 9,78 \\
\hline 190 & 4,76 & 6,34 & 9,51 \\
\hline 200 & 4,64 & 6,18 & 9,27 \\
\hline 210 & 4,52 & 6,03 & 9,05 \\
\hline 220 & 4,42 & 5,89 & 8,84 \\
\hline 230 & 4,32 & 5,77 & 8,65 \\
\hline 240 & 4,23 & 5,64 & 8,46 \\
\hline 250 & 4,15 & 5,53 & 8,29 \\
\hline 260 & 4,07 & $\mathrm{X}$ & 8,13 \\
\hline 270 & 3,99 & $\mathrm{X}$ & 7,98 \\
\hline 280 & 3,92 & $\mathrm{X}$ & 7,84 \\
\hline 290 & $3 ; 85$ & $\mathrm{X}$ & 7,70 \\
\hline 300 & 3,79 & $\mathrm{X}$ & 7,57 \\
\hline
\end{tabular}

TABLEaU III

\begin{tabular}{|c|c|c|c|c|c|c|c|c|c|c|c|}
\hline \multicolumn{4}{|c|}{ Profil $1(k / \beta f=300)$} & \multicolumn{4}{|c|}{ Profil $2(k / \beta \theta=400)$} & \multicolumn{4}{|c|}{ Profil 3 $(k / \beta=600)$} \\
\hline $\begin{array}{c}\text { Charge } \\
\text { HI } \\
\text { en } \\
\text { centi- } \\
\text { mètres }\end{array}$ & $\begin{array}{c}\text { Débit } \\
Q \\
\text { en } \mathrm{l} / \mathrm{s}\end{array}$ & $\begin{array}{c}\text { Volume } \\
\text { empoté } \\
V \\
\text { en } \\
\text { litres }\end{array}$ & $\begin{array}{l}\text { Temps } \\
\text { de } \\
\text { remplis- } \\
\text { sage } \\
\mathrm{T} \\
\text { en } \\
\text { secondes }\end{array}$ & $\begin{array}{c}\text { Charge } \\
\text { H } \\
\text { en } \\
\text { centi- } \\
\text { metres }\end{array}$ & $\begin{array}{c}\text { Débit } \\
Q \\
\text { en } l / s\end{array}$ & $\begin{array}{l}\text { Volume } \\
\text { empoté } \\
\text { V } \\
\text { en } \\
\text { litres }\end{array}$ & $\begin{array}{c}\text { Temps } \\
\text { de } \\
\text { rempis- } \\
\text { suge } \\
T \\
\text { ell } \\
\text { secondes }\end{array}$ & $\begin{array}{l}\text { Charge } \\
\text { H } \\
\text { en } \\
\text { centi- } \\
\text { mètres }\end{array}$ & $\begin{array}{c}\text { Débit } \\
Q \\
\text { en } 1 / \mathrm{s}\end{array}$ & $\begin{array}{l}\text { Volume } \\
\text { empoti } \\
\text { V } \\
\text { en } \\
\text { litres }\end{array}$ & $\begin{array}{c}\text { Temps } \\
\text { de } \\
\text { remplis- } \\
\text { sage } \\
\text { T } \\
\text { en } \\
\text { secondes }\end{array}$ \\
\hline 27,70 & 4,88 & 105,0 & 21,5 & 23,45 & 5,57 & 103,8 & 18,6 & 4,01 & 1,35 & 105 & 77,8 \\
\hline 23,20 & 4,12 & 103,5 & 25,1 & 23,55 & 5,56 & 103,9 & 18,65 & 8,96 & 3,18 & 103,5 & 32,6 \\
\hline 20,30 & 3,62 & 105,2 & 29,1 & 19,30 & 4,68 & 103,5 & 22,1 & 13,78 & 4,91 & 107,5 & 21,9 \\
\hline 16,25 & 2,90 & 102,7 & 35,4 & 19,40 & 4,70 & 103,4 & 22 & 17,32 & 6,0 & 101,5 & 16,9 \\
\hline 12,38 & 2,24 & 104.8 & 46,9 & 17,05 & 4,13 & 104,0 & 25,2 & 25,38 & $8,9(1)$ & (1) & (1) \\
\hline 9,22 & 1,64 & 104,2 & 63,5 & 17,00 & 4,14 & 103,9 & 25,1 & 30,0 & $10,6(1)$ & (1) & (1) \\
\hline 5,94 & 1,05 & 105,4 & 100,5 & 12,90 & 3,18 & 103,3 & 32,9 & 24,82 & $8,9(1)$ & (1) & (1) \\
\hline 2,66 & 0,45 & 103,5 & 230 & 12,85 & 3,18 & 103,8 & 33,1 & 20,30 & $7,5(1)$ & (1) & (1) \\
\hline 6,33 & 1,15 & 105,0 & 91,2 & 9,55 & 228 & 103,6 & 45,4 & 17,32 & 6,05 & 104,0 & 17,2 \\
\hline 8,43 & 1,52 & 101,8 & 66,8 & 9,45 & 2,27 & 103,9 & 45,7 & 11,84 & 4,26 & 101,8 & 23,8 \\
\hline 12,98 & 2,38 & 102,8 & 43,1 & 5,60 & 1,49 & 103,5 & 69,4 & 7,08 & 2,45 & 103,0 & 42 \\
\hline 13,28 & 2,42 & 103,0 & 42,6 & 5,60 & 1,49 & 103,0 & 69 & 4,70 & 1,63 & 102,5 & 62,9 \\
\hline 16,85 & 3,09 & 102,0 & 33 & 2,90 & 0,66 & 106,0 & 160,2 & 3,02 & 1,05 & 105,5 & 103 \\
\hline 17,05 & 3,09 & 102,5 & 33,2 & 2,90 & 0,66 & 106,1 & 161,2 & & & & \\
\hline 21,90 & 3,94 & 103,7 & 26,3 & 2,60 & 0,603 & 103,9 & 172,4 & & & & \\
\hline 27,85 & 4,93 & 109,0 & 22,1 & 2,60 & 0,606 & 104,3 & 172 & & & & \\
\hline 29,35 & 5,21 & 102,0 & 19,6 & & & & & & & & \\
\hline
\end{tabular}

(1) Ces débits ont été mesurés directement au Venturi. 


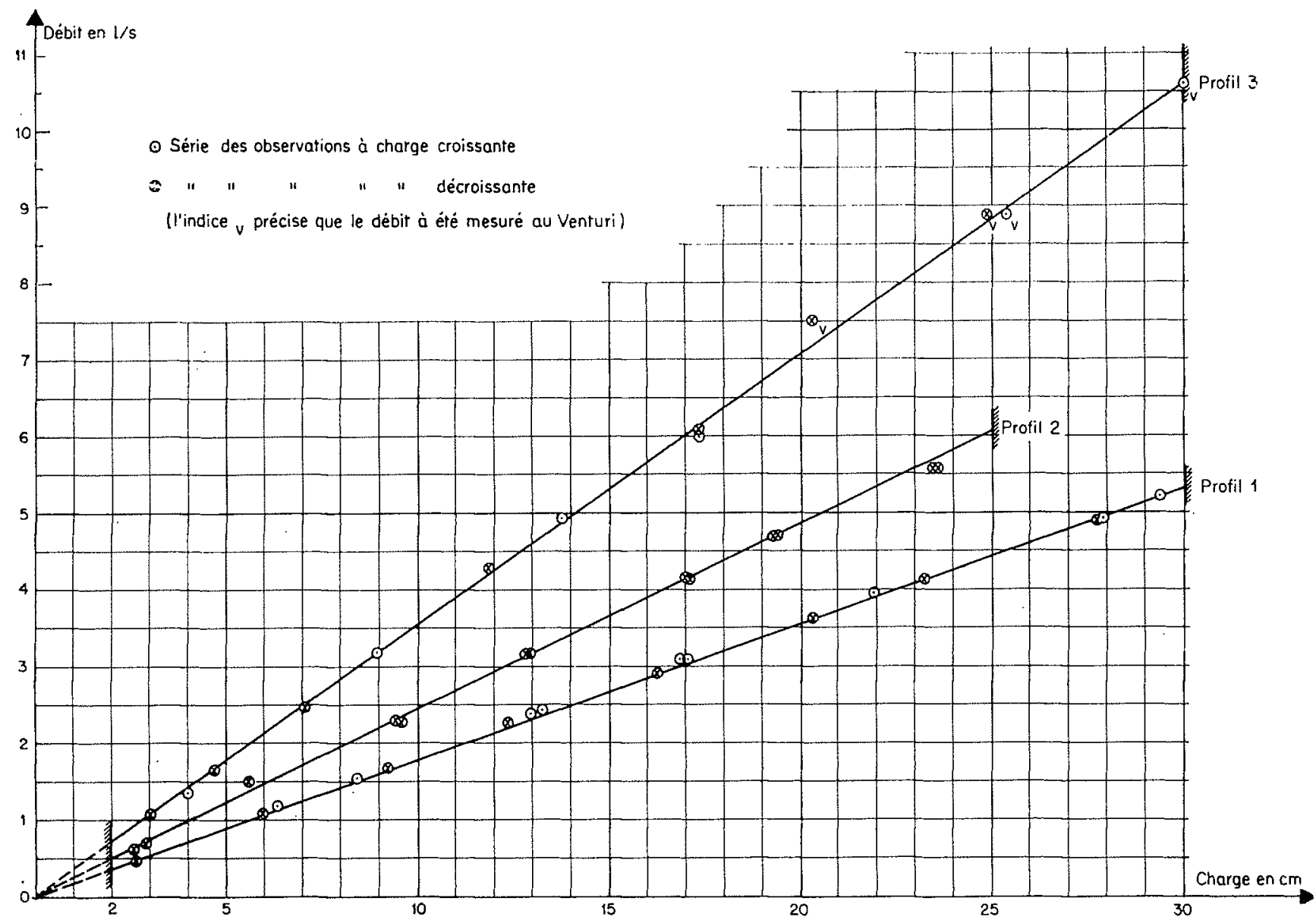

Fig. 21

Etalonnage des plaques déversoirs 1,2 et 3.

Nous pouvons tout d'abord constater que les courbes $\mathrm{Q}(\mathrm{H})$ sont bien des droites d'équation $Q=k H$, et nous pouvons déduire des graphiques les valeurs de $k$ :

$$
\text { - profil } 1 \quad \mathrm{Q}=4,45 \mathrm{l} / \mathrm{s} \quad \begin{aligned}
& \text { pour } \mathrm{H}=25 \mathrm{~cm} \\
& \rightarrow k_{1}=178 \mathrm{~cm}^{2} / \mathrm{s}
\end{aligned}
$$

- profil $2 Q \mathrm{Q}=6,05 \mathrm{l} / \mathrm{s}$ pour $\mathrm{H}=25 \mathrm{~cm}$

$$
\rightarrow k_{2}=242 \mathrm{~cm}^{2} / \mathrm{s}
$$

- profil $3 \mathrm{Q}=8,83 \mathrm{l} / \mathrm{s} \quad$ pour $\mathrm{H}=25 \mathrm{~cm}$

$$
\rightarrow \mathrm{K}_{3}=353 \mathrm{~cm}^{2} / \mathrm{s}
$$

Si nous appelons $Q_{\mathrm{T}}$ le débit théorique satisfaisant à la relation $\mathrm{Q}_{\mathrm{T}}=k \mathrm{H}$, avec les valeurs respectives de $k$ ci-dessus, et si nous appelons $Q_{0}$ le débit observé lors des essais, l'écart absolu $\left|Q_{\mathrm{T}}-Q_{0}\right|$ aura pour limite supérieure théorique :

$$
\left|\Delta Q_{T}\right|+\left|\Delta Q_{0}\right|=k|\Delta H|+\left|\Delta Q_{0}\right|
$$

Considérons d'abord le terme $k|\Delta H|$ : nous avons précisé que $|\Delta \mathrm{H}|=0,5 \mathrm{~mm}$, et, comple lenu des valeurs trouvées pour $k$ nous pouvons dire que :

$$
\begin{aligned}
& \text { profil 1: }\left|\Delta Q_{\mathrm{T}}\right|=8,9 \mathrm{~cm}^{3} / \mathrm{s} ; \\
& \text { profil 2: }\left|\Delta Q_{\mathrm{T}}\right|=12,1 \mathrm{~cm}^{3} / \mathrm{s} ; \\
& \text { profil } 3:\left|\Delta Q_{\mathrm{T}}\right|=17,65 \mathrm{~cm}^{3} / \mathrm{s} .
\end{aligned}
$$

En ce qui concerne le terme $\left|\Delta Q_{0}\right|$ nous devons préciser que $Q_{0}$ est de la forme : V/T, $V$ étant le volume empoté pendant le temps $\mathrm{T}$; dans ces conditions $\left|\Delta \mathrm{Q}_{0}\right|=|\Delta \mathrm{V}| / \mathrm{T}+\mathrm{V}|\Delta \mathrm{T}| / \mathrm{T}^{2}(7)$. Dans la plus grande partie des cas l'on a :

$$
\mathrm{V} \# 105 \text { litres, }
$$

et nous avons déjà précisé que $|\Delta V|=0,2$ litre et $|\Delta T|=0,2$ seconde.

(7) Remarquons que l'erreur relative est :

$$
\left|\Delta Q_{0}\right| / Q_{0}=|\Delta V| / V+|\Delta T| / T
$$


Si nous retenons pour chaque profil les valeurs minimale et maximale de $T$, nous pouvons calculer les valeurs limites correspondantes de $\left|\Delta Q_{0}\right|$ soit :

- profil 1 :

$\left|\Delta Q_{0}\right|$ varie de $62,5 \mathrm{~cm}^{3} / \mathrm{s}(\mathrm{T}=20 \mathrm{~s})$ à $1,3 \mathrm{~cm}^{3} / \mathrm{s}(\mathrm{T}=230 \mathrm{~s})$,

- profil 2:

$\left|\Delta Q_{0}\right|$ varie de $75,9 \mathrm{~cm}^{3} / \mathrm{s}(\mathrm{T}=18 \mathrm{~s})$

$$
\text { à } 1,9 \mathrm{~cm}^{3} / \mathrm{s}(\mathrm{T}=170 \mathrm{~s}) \text {, }
$$

- profil 3 :

$$
\begin{array}{r}
\left|\Delta Q_{0}\right| \text { varie de } 84,5 \mathrm{~cm}^{3} / \mathrm{s} \quad(\mathrm{T}=17 \mathrm{~s}) \\
\text { à } 4,1 \mathrm{~cm}^{3} / \mathrm{s} \quad(\mathrm{T}=100 \mathrm{~s}) .
\end{array}
$$

Nous constatons que, pour les faibles débits c'est l'erreur $k|\Delta \mathbf{H}|$ qui prédomine, alors que pour les débits élevés c'est $\left|\Delta Q_{0}\right|$ qui l'emporte; ceci n'a rien qui doive nous étonner, mais il était intéressant toutefois de le rappeler, ne serait-ce que pour insister sur la diversité des précisions à rechercher selon les conditions de charge, donc de débit.
Dans ces conditions l'écart absolu théorique $\left|\mathrm{Q}_{\mathrm{r}}-\mathrm{Q}_{0}\right|$ variera comme suit :

- profil 1 :

de $71,4 \mathrm{~cm}^{3} / \mathrm{s} \quad(T=20 \mathrm{~s}$, soit $Q \# 5 \quad \mathrm{l} / \mathrm{s})$

à $10,2 \mathrm{~cm}^{3} / \mathrm{s}(\mathrm{T}=230 \mathrm{~s}$, soit $\mathrm{Q} \# 0,45 \mathrm{l} / \mathrm{s})$,

- profil 2:

de $88 \mathrm{~cm}^{3} / \mathrm{s}(\mathrm{T}=18 \mathrm{~s}$, soit $\mathrm{Q} \# 5,8 \mathrm{l} / \mathrm{s})$ à $14 \mathrm{~cm}^{3} / \mathrm{s}(T=170 \mathrm{~s}$, soit $Q \# 0,6 \mathrm{l} / \mathrm{s})$,

- Profil 3 :

de $102,1 \mathrm{~cm}^{3} / \mathrm{s}(T=17 \mathrm{~s}$, soit $Q \# 6 \mathrm{l} / \mathrm{s})$ à $21,7 \mathrm{~cm}^{3} / \mathrm{s}(T=100 \mathrm{~s}$, soit $Q \# 1,05 \mathrm{l} / \mathrm{s})$.

Nous pouvons conclure que, dans l'ensemble, l'erreur absolue varie de 10 à $100 \mathrm{~cm}^{3} / \mathrm{s}$, soit de 0,01 à $0,1 \mathrm{l} / \mathrm{s}$, l'erreur relative étant sensiblement constante et comprise à peu près entre $1,5 \%$ (fortes valeurs de $Q$ ) et 2,5\% (faibles valeurs de $Q$ ).

Si maintenant nous considérons l'écart réel : $Q_{\mathrm{T}}-\mathrm{Q}_{0}$, où $\mathrm{Q}_{0}$ est le débit relevé pour une charge donnée et $Q_{\mathrm{T}}$ le débit théorique, c'est-àdire égal au produit de la dite charge par le

\begin{tabular}{|c|c|c|c|c|c|c|c|c|c|c|}
\hline \multirow{2}{*}{$\begin{array}{c}\text { Profil } 1 \\
k=178 \mathrm{~cm}^{2} / \mathrm{s}\end{array}$} & \multicolumn{2}{|c|}{ Charge en $\mathrm{cm}$} & $\frac{Q_{\mathrm{r}} \mathrm{en}^{\mathrm{cm} / \mathrm{s}}}{4930}$ & $\frac{Q_{0} \text { en } \mathrm{cm}^{3} / \mathrm{s}}{4880}$ & $\begin{array}{l}Q_{r}-Q_{0} \\
+50\end{array}$ & \multicolumn{2}{|c|}{ Charge en cm } & $\frac{\mathrm{Q}_{\mathrm{T}} \text { en } \mathrm{cm}^{3} / \mathrm{s}}{5220}$ & $\frac{Q_{0} \mathrm{en} \mathrm{cm}^{3 / \mathrm{s}}}{5210}$ & $Q_{T}-Q_{0}$ \\
\hline & 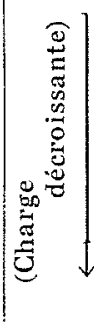 & $\begin{array}{r}27,70 \\
23,20 \\
20,30 \\
16,25 \\
12,38 \\
9,22 \\
594 \\
2,66\end{array}$ & $\begin{array}{r}4930 \\
4130 \\
3615 \\
2890 \\
2205 \\
1640 \\
1057 \\
473\end{array}$ & 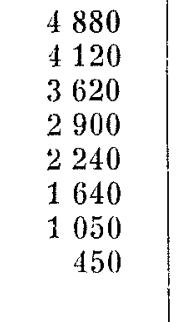 & $\begin{array}{r}+\quad 50 \\
+\quad 10 \\
-\quad 5 \\
-\quad 10 \\
-\quad 35 \\
0 \\
+\quad 7 \\
+\quad 23\end{array}$ & 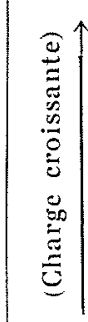 & $\begin{array}{r}29,35 \\
27,85 \\
21,90 \\
17,05 \\
1685 \\
13,28 \\
12,98 \\
8,43 \\
6,33\end{array}$ & 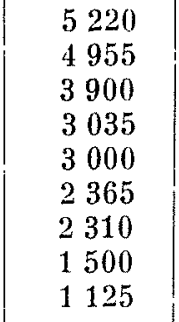 & $\begin{array}{l}5210 \\
4930 \\
3940 \\
3090 \\
3090 \\
2420 \\
2380 \\
1520 \\
1150\end{array}$ & $\begin{array}{l}+\quad 10 \\
+\quad 25 \\
-\quad 40 \\
-\quad 55 \\
-\quad 90 \\
-\quad 55 \\
-\quad 70 \\
-\quad 20 \\
-\quad 25\end{array}$ \\
\hline $\begin{array}{c}\text { Profil } 2 \\
k=242 \mathrm{~cm}^{2} / \mathrm{s}\end{array}$ & 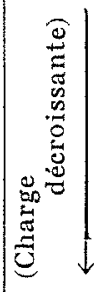 & $\begin{array}{l}23,45 \\
23,55 \\
19,30 \\
19,40 \\
17,05 \\
17,00 \\
12,90 \\
12,85\end{array}$ & $\begin{array}{l}5675 \\
5700 \\
4665 \\
4690 \\
4120 \\
4110 \\
3120 \\
3105\end{array}$ & $\begin{array}{l}5570 \\
5560 \\
4680 \\
4700 \\
4130 \\
4140 \\
3180 \\
3180\end{array}$ & $\begin{array}{r}+105 \\
+140 \\
--\quad 15 \\
-\quad 10 \\
-\quad 10 \\
-\quad 30 \\
-\quad 60 \\
-\quad 75\end{array}$ & 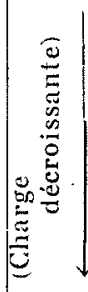 & $\begin{array}{l}9,55 \\
9,45 \\
5,60 \\
5,60 \\
2,90 \\
2,90 \\
2,60 \\
2,60\end{array}$ & $\begin{array}{l}2310 \\
2285 \\
1355 \\
1355 \\
702 \\
702 \\
629 \\
629\end{array}$ & $\begin{array}{r}2280 \\
2270 \\
1490 \\
1490 \\
660 \\
660 \\
603 \\
606\end{array}$ & $\begin{array}{r}+\quad 30 \\
+\quad 15 \\
+\quad 135 \\
+\quad 135 \\
+\quad 42 \\
+\quad 42 \\
+\quad 26 \\
+\quad 23\end{array}$ \\
\hline $\begin{array}{c}\text { Profil } 3 \\
k=353 \mathrm{~cm}^{2} / \mathrm{s}\end{array}$ & 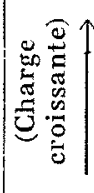 & $\begin{array}{r}17,32 \\
13,78 \\
8,96 \\
4,01\end{array}$ & $\begin{array}{ll}6 & 115 \\
4 & 860 \\
3 & 160 \\
1 & 415\end{array}$ & $\begin{array}{l}6000 \\
4910 \\
3180 \\
1350\end{array}$ & $\begin{array}{r}+115 \\
-\quad 50 \\
-\quad 20 \\
+\quad 65\end{array}$ & 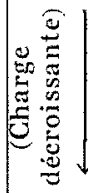 & $\begin{array}{r}17,32 \\
11,84 \\
7,08 \\
4,70 \\
3,02\end{array}$ & $\begin{array}{l}6115 \\
4180 \\
2500 \\
1660 \\
1065\end{array}$ & $\begin{array}{l}6050 \\
4260 \\
2450 \\
1630 \\
1050\end{array}$ & $\begin{array}{r}+\quad 65 \\
+\quad 80 \\
+\quad 50 \\
+\quad 30 \\
+\quad 15\end{array}$ \\
\hline
\end{tabular}
coefficient $k$, nous pouvons dresser le tableau IV.

Tableau IV 
L'examen de ce tableau montre tout d'abord une concordance satisfaisante entre les écarts réels et les écarts calculés. Nous pensons toutefois que les écarts réels sont élevés et nous attribuons ce fait à la trop grande rapidité de nos essais; n'ayant pu consacrer plus d'une journée à chaque essai, nous n'avons pu ni multiplier les mesures de débit, ni utiliser la pointe de lecture au $1 / 10^{\mathrm{e}}$ de $\mathrm{mm}$, dont l'emploi ne se concoit qu'en régime parfaitement stabilisé. Une autre remarque intéressante peut être faite à propos des profils 1 et 3 (le profil 2 ayant été essayé différemment) : les résultats des essais sont meilleurs à charge décroissante qu'à charge croissante; nous pensons que ceci s'explique par la stabilisation du régime que l'on obtient plus rapidement dans le premier cas que dans le second et nous considérons donc qu'il y a lieu de ne faire que des essais à charge décroissante, ce qui par ailleurs permet de doubler pratiquement le nombre de mesures faites dans le même temps.

Les résultats des essais permettent par ailleurs de calculer les coefficients de débit $\theta$, car si l'on considère les profils 1, 2, 3 définis par les valeurs suivantes

$$
\begin{gathered}
(k / \beta \theta)_{1}=300 ; \quad(k / \beta \theta)_{2}=400 \\
\text { et }(k / \beta \theta)_{3}=600,
\end{gathered}
$$

et compte tenu des valeurs trouvées pour $k$, valeurs respectivement égales à $178 \mathrm{~cm}^{2} / \mathrm{s}$, $242 \mathrm{~cm}^{2} / \mathrm{s}$ et $353 \mathrm{~cm}^{2} / \mathrm{s}$ pour les profils 1,2 , et 3 , l'on peut calculer le produit $\beta \theta$, et trouver ainsi :

$$
(\beta \theta)_{1}=0,593 ; \quad(\beta \theta)_{2}=0,605 ; \quad \text { et }(\beta \theta)_{3}=0,588 \text {. }
$$

Il nous reste cependant à séparer l'un de l'autre les coefficients $\beta$ el $\theta$. Ceci n'est guère possible, du moins dans le cadre des essais très rapides que nous avons réalisés, mais nous pouvons considérer que le remplacement par un rectangle de la partie asymptotique à la crête a pour effet un accroissement du coefficient $\beta$ de l'ensemble par rapport à la valeur

$$
\beta=\pi \sqrt{3 / 32} \# 0,962,
$$

correspondant au profil complet, en raisonnant de la manière suivante:

- Si nous considérons un orifice reclangulaire de hauteur $H, h$ étant la charge sur le centre de gravité, nous avons selon Merten (8)

\begin{tabular}{|r|l|l|l|}
\hline pour $h / \mathrm{H}=$ & 0,5 & 0,588 & 1,11 \\
\hline$\beta=$ & 0,944 & 0,964 & 0,99 \\
\hline
\end{tabular}

(8) Voir Cours d'Hydraulique, par L.-J. Tison, tome I, page 108. et, avec nos notations, soit $y_{g}$ la charge sur le centre de gravité du rectangle $\left(y_{y}=y-y_{0} / 3\right)$ et $h_{0}$ la hauteur du rectangle $\left(h_{0}=4 y_{0} / 3\right)$ nous pouvons écrire :

$$
h / \mathbf{H}=y_{g} / h_{0}=\left(3 y-y_{0}\right) / 4 y_{0} ;
$$

comme toutes nos plaques étaient telles que nous avions $y_{11}=2 \mathrm{~cm}$, nous pouvons conclure :

\begin{tabular}{|r|l|l|l|}
\hline$\beta=$ & 0,944 & 0,964 & 0,99 \\
\hline pour $y=$ & $2 \mathrm{~cm}\left(-y_{0}\right)$ & $2,235 \mathrm{~cm}$ & $3,626 \mathrm{~cm}$ \\
\hline
\end{tabular}

- Si nous remarquons maintenant que l'orifice rectangulaire était toujours noyé dans nos essais, nous pouvons écrire $\beta \geqslant 0,964>\pi \sqrt{3 / 32}$. Pour simplifier, nous pouvons écrire : $\beta>0,96$.

Dans ces conditions, le coefficient de débit sera compris entre deux limites définies l'une par $\beta=1$ et l'autre par $\beta=0,96$; ces limites sont alors :

$$
\begin{gathered}
0,593<\theta_{1}<0,618 ; \quad 0,605<\theta_{2}<0,630 \\
\text { et } 0,588<0_{3}<0,612,
\end{gathered}
$$

ce qui permet de dire qu'en moyenne :

$$
0,595<0<0,620 \text {. }
$$

5 "COMPARASON DES RÉsulTats : les résultats de $P$. Morin et les nôtres diffèrent donc très sensiblement puisque :

- selon les résultats de $P$. Morin :

$$
0=m \# 0,75
$$

- selon nos résultats :

$$
0,595<\theta<0,620
$$

Nous passons ci-après en revue les diverses explications possibles, en liaison avec une analyse comparative des conditions d'essais.

\section{A. - Caractéristiques de la paroi et dU} PROFIL

Les déversoirs étaient dans les deux cas en paroi mince : plaques de $2 \mathrm{~mm}$ d'épaisseur avec profils à bord droit. Les écoulements étaient de plus dénoyés.

Dans nos essais la face aval seule était polie, la face amont étant restée brute (ou du moins dans l'ctat naturel du laiton). Dans les essais de 
P. Morin, la face amont n'était certainement pas d'une rugosité particulièrement élevée (cannelures, grains de quartz collés, etc.) et nous pouvons donc penser que la surface du duralumin était soit polie, soit plus probablement brute. Etant donné que la rugosité de la face amont accroît le débit, nous pouvons admettre que nos résultats sont au moins comparables à ceux de $P$. Morin dans la première hypothèse (faces amont brutes dans les deux cas), sinon supérieurs dans la deuxième hypothèse (face amont brute dans nos essais et polie dans ceux de P. Morin).

Si l'on considère le rapport $\rho$ de l'aire $\Omega_{0}$ du rectargle (équivalent) à l'aire totale $\Omega(y)$, et compte tenu de la relation :

$$
?=\Omega_{0} / \Omega=x / x_{0}=\sqrt{y_{0} / y}
$$

nous pouvons observer que ces rapports sont plus élevés dans nos expériences que dans celles de P. Morin. Nous avions en effet, pour les essais de $P$. Morin : $?=0,158$ avec la plaque $\& A »$ et $\wp=0,174$ pour la plaque $\ll B \gg$; ces valeurs étaient plus élevées dans nos essais car nous avions : $?=0,257$ pour les profils 1 et 3 , et $p=0,315$ pour le profil 2. Or lorsque $\Omega_{0} / \Omega$ augmente, le rapport $h / H$ défini précédemment diminue, ainsi que le coefficient $\beta$ par voie de conséquence; dans ces conditions le coefficient $\beta$ présentait des valeurs plus faibles pour nos essais que pour ceux de P. Morin et nous pourons considérer que le coefficient $\theta$ était au contraire plus élevé dans nos essais que dans ceux de P. Morin, toutes choses étant égales d'ailleurs.

Enfin nous avons, dans tout ce qui précède, considéré des déversoirs placés en paroi latérale verticale, cas auquel correspond le coefficient $\beta$. Si la paroi fait au contraire un angle $\alpha$ avec la verticale, le coefficient devient $\beta^{\prime}$ tel que : $\beta^{\prime}=\beta / \cos \alpha$, c'est-à-dire que nous avons $\beta^{\prime}>\beta$; or la photo 4 du mémoire de $P$. Morin [9] révèle l'existence d'un tel défaut de verticalité, qui pour être faible, n'en contribue pas moins à accroître le coefficient $\beta$, donc à diminuer le coefficient $\theta$, dans le cas de P. Morin.

\section{B. - Ciractéristiques des dispositers d'essai}

La comparaison des deux dispositifs est en fait particulièrement délicate, car ceux-ci sont fondamentalement différents; une analyse des conditions d'essai permet de faire les constatations suivantes :

\section{a) En ce qui concerne le champ des vitesses :}

Dans les essais de P. Morin, l'alimentation du bac de mesure se faisant par le fond, les vitesses peuvent conserver une composante verticale non négligeable et nous en voyons la preuve dans les remous superficiels qui apparaissent sur une photographie faisant partie de l'étude citée [9]. Ce phénomène entraîne un accroissement des vitesses superficielles, d'où résulte un accroissement du débit.

Dans nos essais le dispositif se rapproche beaucoup d'un déversoir Rehbock. Orr de nombreuses études, et en particulier celles de Rehbock, ont permis de constater que, si les résultats de Bazin pouvaient être considérés comme généralement trop élevés, de 1 à $5 \%$ selon les auteurs et les conditions, l'écart devait être attribué aux grandes vitesses superficielles réalisées dans les déversoirs Bazin, en canal long (23 mètres); au contraire les autres auteurs, dont Rehbock, travaillaient plus généralement avec des canaux courts (moins de $7 \mathrm{~m}$ ) et utilisaient des dispositifs d'uniformisation des vitesses. Dans ces conditions, et considérant que le dispositif de $P$. Morin favorise particulièrement les vitesses superficielles, nous verrons dans la différence de répartition des vitesses à l'amont du déversoir une explication des écarts enregistrés.

\section{b) En ce qui concerne la hauteur de pelle:}

Dans les essais de $P$. Morin, la hauteur de pelle $p$ était certainement supérieure à la charge $h$ la plus forte; dans ces conditions, nous pouvons dire que le rapport $h_{i}(h+p)$ était compris entre 0 et 0,5 au maximum.

Dans nos essais, nous avions $p=42$ à $47 \mathrm{~mm}$, et finalement le rapport $h /(h+p)$ était compris entre 0 et 0,83 pour le profil 2 , entre 0 et 0,88 pour les profils 1 et 3 .

Or toutes les expériences montrent que le coefficient de débit augmente avec $h /(h+p)$. Il ressort de ceci que nos essais devaient, toutes choses étant égales d'ailleurs, nous donner des résultats plus élevés que ceux de P. Morin, c'esta-dire que, si les conditions avaient été les mêmes quant à la hauteur de pelle, l'écart aurait été encore plus grand entre les valeurs du coefficient de débit.

\section{c) En ce qui concerne les dimensions latérales respectives de l'échancrure et du dispo- sitif d'essai :}

Il a été démontré que, pour un déversoir rectangulaire de largeur $l$ placé dans un canal de largeur $L$, le coefficient de débit augmentait avec le rapport $l / L$. 
Les déversoirs essayés par $P$. Morin et par nous-mêmes ayant une largeur $l$ variable, mais toujours nettement inférieure à la largeur constante $L$ du canal ou du bac, nous pouvons penser par analogie que les conditions d'essais étaient dans les deux cas de nature à réduire le coefficient de débit; précisons que la largeur $\mathrm{L}$ du bac utilisé par P. Morin était du même ordre de grandeur que celle du canal utilisé pour nos essais.

\section{C. - Considérations de similitude}

Dans la théorie des écoulements à surface libre (orifices et déversoirs), et en particulier d'après les lois de la similitude, l'on démontre qu'il est possible d'obtenir des courbes d'égal coefficient de débit $\theta$ en fonction du nombre de Reynolds $\mathcal{R}$ et du rapport $v / \mathrm{H}^{3 / 2}$, où $v$ est la viscosité cinématique et $\mathrm{H}$ la charge.

Dans le nombre de Reynolds $\mathcal{R}=v d / v$, nous pouvons prendre, au lieu de la vitesse réelle $v$ dans la section contractée, vitesse difficilement mesurable, la valeur $\sqrt{2 g H}$, puisque, dans de tels systèmes, la loi de Reech-Froude doit être aussi vérifiée, ce qui revient à poser

$$
\frac{v}{\sqrt{g \mathrm{H}}}=\text { Cte }=\mathscr{g}(\mathscr{H}=\text { nombre de Froude })
$$

Les deux séries d'essais ayant été faites avec de l'eau et à la température ambiante, les variations de $v$ sont pratiquement négligeables, d'un essai à l'autre.

Par contre, et pour toutes les raisons exposées précédemment, les essais ont été faits dans des conditions tellement différentes que nous ne pouvons guère envisager d'utiliser l'ensemble des résultats en vue de tracer les courbes d'égale valeur de 0 ; de plus, il y a lieu de remarquer que, pour une étude de ce genre, la précision des mesures doit être maximale, ce qui n'était pas absolument nécessaire pour nous, compte tenu du but recherché (démonstration de la linéarité de $Q(H)$ et recherche de la valeur moyenne de $\theta$ ).
Nous pouvons toutefois remarquer que la plus forte valeur de $\theta$ observée l'a été avec le profil 2, alors même que c'est ce profil qui a été essayé avec la charge maximale la plus faible. Ce fait pourrait correspondre à la loi bien établie selon laquelle le coefficient de débit varie en sens inverse de la charge. Il ne faut cependant pas négliger le fait que le rapport $\rho=\Omega_{0} / \Omega$ vaut 0,315 pour le profil 2 , et seulement 0,257 pour les profils 1 et 3 , ce qui entraîne un coefficient $\beta$ plus faible, donc un coefficient de débit $\theta$ plus élevé; nous ne pourons donc pas savoir si cet accroissement de $\theta$ pour le profil 2 est dû soit à la faible charge $H$, soit à la forte valeur du rapport $\rho$, l'hypothèse la plus probable étant que ces deux phénomènes jouent simultanément.

$$
\text { D. Conclusions }
$$

Nous pouvons affirmer que l'écart entre les résultats de $P$. Morin et les nôtres est certainement supérieur à celui qui existe entre les valeurs

$$
\theta=0,75 \quad \text { et } \theta=0,62 \text {, }
$$

d'une part en raison de ce que, dans les essais de P. Morin, ce n'est pas en fait $\theta$ qui est déterminé, mais bien le produit $\beta \theta$; comme nous avons $\beta<1$ nous pouvons écrire :

$$
\theta=0,75 / \beta>0,75
$$

- D'autre part, et pour toutes les raisons développées aux $\S \S$ A et $B$, nous pouvons affirmer que nos essais ont été faits dans des conditions propres à accroître le coefficient de débit $\theta$ : si nous nous mettions dans les conditions des essais de $P$. Morin, nous ne pourrions trouver que des valeurs inférieures de $\theta$ et nous pouvons donc écrire $\theta<0,62$.

Nous pouvons donc conclure que l'écart entre les résultats est certainement supérieur à $21 \%$ $[=(0,75-0,62) / 0,62]$ et plus vraisemblablement de l'ordre de $25 \%$. De tels écarts sont signalés comme possibles par L. J. Tison qui les attribue aux accroissements des vitesses superficielles, explication que nous retiendrons, et qui attire une fois de plus l'attention sur l'importance bien connue des conditions d'essais. 


\section{CONCLUSIONS GENERALES}

Sur le plan théorique le plus général, nous avons démontré que toutes les formules, parfois très différentes d'expression, étaient en fait strictement identiques, sous réserve de modifications généralement peu importantes, ou du moins non fondamentales à apporter à chacune d'elles afin de tenir compte des différences existant entre les hypothèses de base. Cette unité ne doit pas nous étonner, mais elle nous autorise à préférer, le fond étant le même, la forme des expressions de P. Morin et L. Hugues, en raison même de leur simplicité; pour les mêmes raisons, nous préconisons le remplacement de la partie asymptotique de $y=\mathrm{A} / x^{2}$ par un orifice circulaire.

Aux travaux cités, il convient d'ajouter pour être complet, ceux de Von Rother [11], [12] dont nous n'avons que les références, empruntées à une note résumée de P. Morin [14]. Nous pouvons donc penser que, sous réserve de vérifications en ce qui concerne les travaux de Von Rother, le bénéfice de l'antériorité puisse être reconnu aux travaux de G. Di Ricco. Enfin, nous ne devons pas oublier l'étude de S. Barocio Barrios [15] qui développe une méthode de différences finies pour déterminer le profil correspondant à une courbe expérimentale $Q(\mathrm{H})$ n'ayant pas d'expression analytique; cette méthode s'applique surtout aux problèmes posés par l'étude sur modèle réduit d'un ouvrage en rivière et tout particulièrement à la réalisation, pour le modèle, de la même loi hauteur-débit que dans la nature.

Sur le plan expérimental, et plus particulièrement en ce qui concerne la détermination du coefficient de débit $\theta$, nous avons démontré que les déversoirs en «Tour Eiffel» qui, si l'on en jugeait par les résultats de $P$. Morin $(\theta \# 0,75)$, se distinguaient nettement des Sutro-Weirs $(\theta \# 0,62)$, ne s'écartent de ceux-ci qu'en raison de conditions d'essais très particulières et que, pour des essais plus classiques en canal Reh- bock, le coefficient de débit 0 des déversoirs en «Tour Eiffel» n'est guère éloigné des valeurs observées pour les Sutro-Weirs.

Sur le plan des applications pratiques, nous pouvons préciser que, s'il s'agit de mesures de débits d'eaux courantes, l'essai en canal Rehbock est certainement plus proche des conditions naturelles que l'essai en bac de P. Morin. Par contre, lorsqu'il s'agit de mesures de débits de captages, il semble que l'essai en bac de $P$. Morin doive être plus proche de la réalité, soit que le captage se trouve naturellement dans des conditions comparables à celles de l'essai (par exemple griffon capté par simple dégagement, puis couverture de l'émergence, avec remous superficiels près de la plaquedéversoir), soit que le captage puisse recevoir aux moindres frais un aménagement simple destiné à reproduire les conditions d'essai (construction d'un petit bac de mesure alimenté par le captage proprement dit). Dans les prospections, nous devrons donc, compte tenu des écarts importants existants entre les deux courbes $\mathrm{Q}(\mathrm{H})$, examiner avec soin les conditions naturelles de chaque mesure, en vue de déterminer la courbe $\mathrm{Q}(\mathrm{H})$ qui correspond le mieux à ces conditions, ainsi que les aménagements pouvant éventuellement être apportés afin d'ajuster du mieux possible les conditions de l'essai sur le terrain à celles de l'essai en laboratoire. La double gravure, une courbe $Q(H)$ sur chaque lace de la plaque, permet de limiler le nombre de plaques.

Nous pouvons donc dire en conclusion que de nombreuses études tant théoriques qu'expérimentales peuvent êtes faites dans le domaine des déversoirs en «Tour Eiffel». En particulier nous pensons qu'il serait intéressant d'étudier la variation du coefficient de débit 0 en fonction du seul paramètre $A$, dans le cas de la section équivalente circulaire.

\section{Remerciements}

Nous remercions tout particulièrement $M$. P. Laffitte, Directeur Général Adjoint du B.R.G.M. qui a bien voulu nous autoriser à publier notre étude, M. C. Beaumont, Directeur Scientifique du B.R.G.M., et M. G. Castany, Chef du Département Géologique du B.R.G.M., qui nous ont permis de mener à bien nos recherches.

Nos remerciements vont aussi à $M$. P. Becker,
Ingénieur Général du Génie Rural, Directeur de l'Ecole Nationale du Génie Rural, qui nous a autorisé à utiliser les installation du Laboratoire d'Hydraulique de l'Ecole; nous remercions enfin M. Carlier, Ingénieur en Chef du Génie Rural, Professeur d'Hydraulique de l'E.N.G.R., qui a bien voulu nous donner quelques conseils, que ce soit au Laboratoire ou dans son bureau. 


\section{BIBLIOGRAPHIE}

[1] PRATT E.A. - Another proportional-flow weir; Sutro-Weir, Engineering News, August 27, 1914.

(2) Soucek E., Howe H.E., and Mavis F. T. - SutroWeir investigations furnish discharge coefficients. Engineering News-Record, November 12, 1936.

[3] SMLTH E.G. - A weir with flow proportional to head. Engineering News-Record, July 29, 1943.

[4] Lincoln R.A. - Inflltration measurements in sewers of small size. Journal of the Sanitary Engineering Division, Proc. of the Am. Soc. of Civ. Eng., April 1957.

[5] Dr Ricco G. - Equazione di forma di uno stramazzo dedotta dalla sua equazione di portata. L'Energin elethica, ottobre 1936.

[6] Dr Ricco G. - Stramazzi con data equazione di portata. L'Energin elettrica, Febbraio 1939.

[7] DI Ricco G. - Edificio di misura ad equazione di portata lineare. Rivista del Catasfo e dei Servizi tecnici erariali, n' 3 e 4, 1940.

[8| Dr Ricco G. - Edificio di misura ad equazione di portata lineare. Funzionamento sotto rigurgito.
Rivista del Catasto e dei Servizi tecnici erariali, $\mathrm{n}^{\circ} 1,1949$.

[9] Mon $P$. - Déversoir à variations linéaires et sensibilité constante. Annales de la Direction générale du Génie rural et de l'Hydraulique agricole (Ministère de l'Agriculture), fascicule 73, 1953.

[10] Huaues L. -- Détermination théorique du profil d'un déversoir en paroi mince réalisant une loi de débit imposée. Comptes rendus de l'Académie des Sciences de Paris, tome 242, $\mathrm{n}^{\circ} 16,16$ avril 1956.

[11] von Rother. - Der proportional Ueberfall. Wasser und Gas, n"S-1216, 1921.

[12] VoN RотнER. - Der kreisrunde Ueberfall und seino Abarten. Wusser und Gas, 1926.

13) Roubret P. A. - Le déversoir à loi linéaire. Mécanique, 1942 , p. 258.

[14] Monin P. - Déversoil à variations linéaires et sensibilité constante. $L^{\prime} E a t h, 42^{\circ}$ année, $n^{\circ} 8$, aont 1955.

[15] Baroga Barros S. - La détermination d'un déversoir devant respecter une loi hauteur-débit donnéc. La Houille Blanche, $\mathrm{n}^{\circ} 4$ août-septembre 1953.

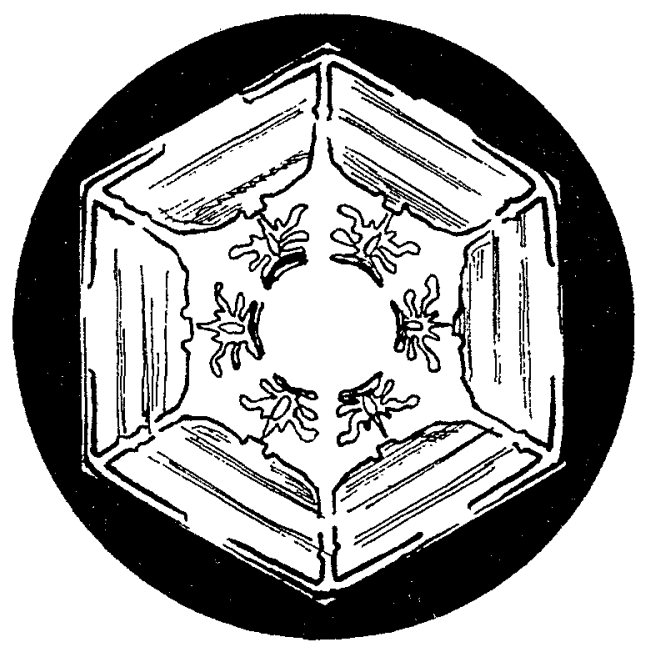

